\title{
Quantum consistency in supersymmetric theories with R-symmetry in curved space
}

\author{
Ok Song An, ${ }^{a}$ Jin U. Kang, ${ }^{b, a}$ Jong Chol Kim ${ }^{a}$ and Yong Hae Ko ${ }^{a}$ \\ ${ }^{a}$ Department of Physics, Kim Il Sung University, \\ Ryongnam Dong, TaeSong District, Pyongyang, DPR Korea \\ ${ }^{b}$ International Centre for Theoretical Physics, \\ Strada Costiera 11, Trieste 34151, Italy \\ E-mail: an.oksong@outlook.com, jkang@ictpt.it, kimjongchol2@gmail.com, \\ yonghae.ko@outlook.com
}

Abstract: We discuss consistency at the quantum level in the rigid $\mathcal{N}=1$ supersymmetric field theories with a $\mathrm{U}(1)_{R}$ symmetry in four-dimensional curved space which are formulated via coupling to the new-minimal supergravity background fields. By analyzing correlation functions of the current operators in the $\mathcal{R}$-multiplet, we show that the quantum consistency with the (unbroken) supersymmetry requires the $\mathrm{U}(1)_{R}$ anomaly coefficient, which depends only on the field content of the theory, to vanish. This consistency condition is obtained under the assumption that the supercurrent Ward identity is non-anomalous and that the vacuum is supersymmetric.

KEYwords: Anomalies in Field and String Theories, Supersymmetric Effective Theories, Supergravity Models, Supersymmetric Gauge Theory

ArXiv EPrint: 1902.04525 


\section{Contents}

1 Introduction 1

2 New minimal supergravity and Killing spinor 3

3 Ward identities and correlation functions 5

3.1 Ward identities 5

$\begin{array}{ll}3.2 & \text { Higher-point correlation functions } \\ \end{array}$

4 Rigid supersymmetry algebra $\quad 9$

5 Quantum consistency 13

$\begin{array}{lll}6 & \text { Discussions } & 16\end{array}$

$\begin{array}{ll}\text { A Conventions } & 17\end{array}$

B An example: $\mathcal{N}=1$ with a free chiral multiplet on $\mathbb{R} \times S_{r_{3}} \quad 18$

\section{Introduction}

Supersymmetric field theories in curved space have attracted many interests in recent years. For such theories supersymmetric localization technique [1, 2] allows the non-perturbative exact computation of some interesting physical quantities such as the partition function and BPS Wilson loops, which can be used to test the duality conjectures like the AdS/CFT correspondence [3-5]. In this paper we focus on supersymmetric field theories with a $\mathrm{U}(1)_{R}$ symmetry on curved manifolds in $3+1$ dimensions.

According to [6-8] (see also [9] for a recent review), one can formulate a $\mathcal{N}=1$ theory with a $\mathrm{U}(1)_{R}$ symmetry in $4 \mathrm{D}$ curved space via coupling to the new-minimal supergravity $[10,11]$ : one first couples the $\mathcal{R}$-multiplet to the new-minimal supergravity multiplet and then take a rigid limit sending the Newton's constant to zero, so that the supergravity is decoupled while the fields in the supergravity multiplet are sent to fixed backgrounds. In constructing supersymmetric field theories in curved space, background fields are typically chosen to be bosonic, and consistency with supersymmetry requires the supersymmetry variation of the gravitino in the gravity multiplet to vanish, which leads to a generalized Killing spinor (GKS) equation. For each solution of the GKS equation there exists a conserved supercharge. In the case of $4 \mathrm{D} \mathcal{N}=1$ theory with a $\mathrm{U}(1)_{R}$ symmetry, the GKS equation can have a solution if and only if the background manifold admits an integrable complex structure and a compatible Hermitian metric.

Important exact results were obtained for the $4 \mathrm{D} \mathcal{N}=1$ supersymmetric field theory with an $R$-symmetry in [12-17], using localization technique. Here we list some of them: 
- When there are two supercharges with opposite $\mathrm{U}(1)_{R}$ charge, supersymmetric renormalization scheme is free of ambiguities and the partition function is invariant under the deformation of the Hermitian metric for a fixed complex structure.

- When the background manifold is $S_{r_{3}}^{3} \times S_{\beta}^{1}$, the supersymmetric Casimir energy becomes

$$
E_{\text {susy }} \equiv-\lim _{\beta \rightarrow \infty} \frac{\mathrm{d}}{\mathrm{d} \beta} \log Z_{\text {susy }}=\frac{4}{27 r_{3}}(a+3 c),
$$

where $a$ and $c$ are two trace anomaly coefficients in four dimensions.

We recall that the field theory computations in [12-17] were carried out by using the supersymmetric Ward identities and the supersymmetry algebra which are derived from the classical new-minimal supergravity. In [18] (see also section 7.10 of [19]) it was argued via the superspace formalism that the new-minimal supergravity can be in general quantummechanically inconsistent, due to the appearance of the "superscale" anomalies (consisting of the conformal anomaly, the $\mathrm{U}(1)_{R}$ chiral anomaly and the $\gamma$-trace of the supercurrent) that are inconsistent with the local supersymmetry Ward identities. Then one could also question the quantum consistency of the new-minimal supergravity formulation of the rigid $\mathcal{N}=1$ field theories with an $R$-symmetry. However, [18] considered only the one-point function of the supercurrent superfield and one could expect that there is no inconsistency for the backgrounds on which the anomalies in the Ward identities (for current one-point functions) are numerically vanishing. In particular, the backgrounds considered in [14$17,20,21]$ are anomaly-free in this sense. Nevertheless, the anomalies might appear in the higher-point correlation functions as the contact terms, some of which may be physically meaningful. This motivates us to study the higher-point correlation functions to investigate the quantum consistency.

In this paper we analyze the two-point and higher-point correlation functions of the current operators in the $\mathcal{R}$-multiplet. Assuming that the supercurrent Ward identity is non-anomalous and that the vacuum is supersymmetric, we show that the unbroken supersymmetry is inconsistent at the quantum level unless the coefficient of the $\mathrm{U}(1)_{R}$ anomaly vanishes. Note that the anomaly coefficients depend only on the field content of the theory. Since the Ward identities and the rigid supersymmetry algebra are a direct consequence of the new-minimal supergravity, this implies that the $4 \mathrm{D} \mathcal{N}=1$ field theory with an $R$-symmetry can be consistently formulated in terms of the new-minimal supergravity only for some special systems with the field content that leads to vanishing coefficient of $\mathrm{U}(1)_{R}$ anomaly. One example of this is a theory that consists of a free chiral multiplet with $R$-charge 1 , since in this case the Weyl fermion in the chiral multiplet is uncharged under the $\mathrm{U}(1)_{R}$ symmetry and gives no contribution to the $\mathrm{U}(1)_{R}$ chiral anomaly.

The rest of this note is organized as follows. In section 2 we briefly review the symmetries of the new-minimal supergravity, the definition of the generalized Killing spinor (GKS) and the construction of the Killing vector from the GKSs. We then derive the Ward identities of $\mathcal{N}=1$ supersymmetric field theories with an $R$-symmetry in section 3 , the results of which are used to reproduce the rigid supersymmetry algebra in section 4 . In section 5 we show that in order for $\mathcal{N}=1$ field theories with an $R$-symmetry to be consistently 
formulated via the new-minimal supergravity the (pure) $\mathrm{U}(1)_{R}$ chiral anomaly coefficient should vanish. Finally, we end with concluding remarks in section 6. Appendix A contains our conventions, while appendix B explicitly derives the transformation laws of the current operators in $\mathcal{R}$-multiplet in a $\mathcal{N}=1$ supersymmetric theory with a free chiral multiplet on $\mathbb{R} \times S^{3}$.

\section{New minimal supergravity and Killing spinor}

In this section, we briefly review the symmetries of the new-minimal supergravity and define a GKS and a Killing vector, as preliminaries for deriving the Ward identities of the $\mathcal{N}=1$ field theory with an $R$-symmetry in the next section.

We begin with the construction of the new-minimal supergravity $[10,11]$. It is formulated by first minimal-coupling the $\mathcal{R}$-multiplet (consisting of energy-momentum tensor $\mathcal{T}^{\mu \nu}$, supercurrent $\mathcal{S}_{\alpha}^{\mu}, \mathrm{U}(1)_{R}$ current $\mathcal{J}^{\mu}$ and closed two-form $\left.\mathcal{F}_{\mu \nu}\right)$ to the supergravity multiplet (containing metric $g_{\mu \nu}$, gravitino $\psi_{\alpha \mu}, \mathrm{U}(1)_{R}$ gauge field $A_{\mu}$ and two-form gauge field $B_{\mu \nu}$ ) to obtain the linear Lagrangian, which is then completed to the non-linear form. The linear couplings take the form

$$
-\frac{1}{2} \mathcal{T}^{\mu \nu} g_{\mu \nu}+\bar{\psi}_{\mu} \mathcal{S}^{\mu}+\mathcal{J}^{\mu}\left(A_{\mu}-\frac{3}{2} V_{\mu}\right)+\frac{1}{4} \varepsilon^{\mu \nu \rho \lambda} \mathcal{F}_{\mu \nu} B_{\rho \lambda},
$$

where $V_{\mu}=\frac{1}{4} \varepsilon_{\mu}{ }^{\nu \rho \lambda} \partial_{\nu} B_{\rho \lambda}$. By definition, the vector field $V^{\mu}$ is conserved, i.e. $\nabla_{\mu} V^{\mu}=0$. In general backgrounds the operators in the $\mathcal{R}$-multiplet are defined in terms of the functional derivatives of the action $S$ with respect to corresponding fields in the supergravity multiplet, namely

$$
\begin{aligned}
\mathcal{T}^{\mu \nu}(x) & =-\frac{2}{e} \frac{\delta S}{\delta g_{\mu \nu}(x)}, \\
\mathcal{S}^{\mu}(x) & =\frac{1}{e} \frac{\delta S}{\delta \bar{\psi}_{\mu}(x)}, \\
\mathcal{J}^{\mu}(x) & =\frac{1}{\boldsymbol{e}} \frac{\delta S}{\delta A_{\mu}(x)}, \\
\mathcal{F}_{\mu \nu}(x) & =\frac{1}{e} \varepsilon_{\mu \nu \rho \lambda} \frac{\delta S}{\delta B_{\rho \lambda}(x)},
\end{aligned}
$$

where $\boldsymbol{e} \equiv\left|\operatorname{det} e_{\mu}^{a}\right|$ with $e_{\mu}^{a}$ being the vierbein.

At the classical level the new-minimal supergravity possesses the local supersymmetry as well as the $\mathrm{U}(1)_{R}$ chiral symmetry and the diffeomorphism invariance. The corresponding transformation laws for the fields in the supergravity multiplet are given as follows (for conventions see appendix A):

- Local supersymmetry transformation

$$
\begin{aligned}
\delta_{\epsilon} e_{\mu}^{a} & =-\frac{1}{2} \bar{\psi}_{\mu} \gamma^{a} \epsilon, \\
\delta_{\epsilon} \psi_{\mu} & =\mathcal{D}_{\mu} \epsilon+\frac{i}{2} \gamma_{\mu}\left(V^{\rho} \gamma_{\rho} \gamma_{*} \epsilon\right),
\end{aligned}
$$




$$
\begin{aligned}
\delta_{\epsilon} B_{\mu \nu} & =\frac{1}{2}\left(\bar{\psi}_{\mu} \gamma_{\nu}-\bar{\psi}_{\nu} \gamma_{\mu}\right) \epsilon \\
\delta_{\epsilon} A_{\mu} & =\frac{i}{4}\left(\mathcal{D}_{\lambda} \bar{\psi}_{\sigma}+\frac{i}{2} \bar{\psi}_{\sigma} \gamma_{*} \gamma_{\kappa} V^{\kappa} \gamma_{\lambda}\right) \gamma_{*} \gamma^{\lambda \sigma} \gamma_{\mu} \epsilon .
\end{aligned}
$$

- $\mathrm{U}(1)_{R}$ chiral gauge transformation

$$
\begin{aligned}
& \delta_{\Lambda} A_{\mu}=\partial_{\mu} \Lambda, \\
& \delta_{\Lambda} \psi_{\mu}=-i \gamma_{*} \psi_{\nu} \Lambda .
\end{aligned}
$$

- Diffeomorphism

$$
\begin{aligned}
\delta_{\xi} g_{\mu \nu} & =\mathcal{L}_{\xi} g_{\mu \nu}=\nabla_{\mu} \xi_{\nu}+\nabla_{\nu} \xi_{\mu} \\
\delta_{\xi} A_{\mu} & =\mathcal{L}_{\xi} A_{\mu}=-F_{\mu \nu} \xi^{\nu}+\nabla_{\mu}\left(\xi^{\nu} A_{\nu}\right) \\
\delta_{\xi} B_{\rho \lambda} & =\mathcal{L}_{\xi} B_{\rho \lambda}=\xi^{\kappa} \nabla_{\kappa} B_{\rho \lambda}+\nabla_{\rho} \xi^{\kappa} B_{\kappa \lambda}+\nabla_{\lambda} \xi^{\kappa} B_{\rho \kappa}, \\
\delta_{\xi} \psi_{\mu} & =\xi^{\nu} \partial_{\nu} \psi_{\mu}+\left(\partial_{\mu} \xi^{\nu}\right) \psi_{\nu} .
\end{aligned}
$$

Here $F_{\mu \nu} \equiv \nabla_{\mu} A_{\nu}-\nabla_{\nu} A_{\mu}$. We have omitted the higher-order terms in the gravitino, since they are irrelevant to our analysis. One can confirm the above transformation laws by checking invariance of the pure new-minimal supergravity action, given by

$$
S_{\mathrm{nm}}=\frac{1}{2} \int \mathrm{d}^{4} x \boldsymbol{e}\left(R+6 V_{\mu} V^{\mu}-8 A_{\mu} V^{\mu}-\bar{\psi}_{\mu} \gamma^{\mu \nu \rho} \mathcal{D}_{\nu} \psi_{\rho}+(4 \text { fermion terms })\right),
$$

where $R$ is a Ricci scalar.

As mentioned in the Introduction, a field theory with rigid supersymmetry is defined on the supersymmetric backgrounds, i.e. the ones that admit at least one solution of the GKS equation. For $\mathcal{N}=1$ field theories with an $R$-symmetry the GKS equation becomes

$$
\delta_{\epsilon} \psi_{\mu}=\mathcal{D}_{\mu} \epsilon+\frac{i}{2} \gamma_{\mu}\left(V^{\rho} \gamma_{\rho} \gamma_{*} \epsilon\right)=0 .
$$

Now let us denote a solution of $(2.7)$ as $\zeta$. As we will see in section 3 , there exists a conserved supercharge corresponding to $\zeta$. For the discussion of the supersymmetry algebra given in section 3 , we need to define a real vector ${ }^{1}$ (i.e. $K^{*}=K$ )

$$
K=K^{\mu} \partial_{\mu} \quad \text { with } \quad K^{\mu}=\bar{\eta} \gamma^{\mu} \zeta .
$$

Here $\eta \equiv i \gamma_{*} \zeta$ is also a GKS. By using the Fierz identity and the integrability condition for the GKS $\zeta$, one can derive following relations [22]:

$$
\begin{aligned}
K^{\mu} \gamma_{\mu} \zeta & =0, \quad K^{\mu} K_{\mu}=0, \\
\mathcal{L}_{K} \zeta & =K^{\mu} \nabla_{\mu} \zeta+\frac{1}{4} \nabla_{\mu} K_{\nu} \gamma^{\mu \nu} \zeta=-K^{\mu} A_{\mu}\left(i \gamma_{*} \zeta\right), \\
\nabla_{\mu} K_{\nu} & =-\varepsilon_{\mu \nu \rho \sigma} V^{\rho} K^{\sigma}, \quad K^{\mu} \nabla_{\mu} V_{\nu}=0, \\
F_{\mu \nu} K^{\mu} & =0 .
\end{aligned}
$$

\footnotetext{
${ }^{1}$ Notice that $\bar{\epsilon} \gamma_{\mu} \epsilon=0$, which follows from the property of the Majorana conjugation.
} 
It therefore follows that the background fields are invariant with respect to the null vector $K$ up to a gauge transformation for the $\mathrm{U}(1)_{R}$ gauge field $A$, namely

$$
\begin{aligned}
\mathcal{L}_{K} g_{\mu \nu} & =\nabla_{\mu} K_{\nu}+\nabla_{\nu} K_{\mu}=0, \\
\mathcal{L}_{K} A_{\mu} & =-F_{\mu \nu} K^{\nu}+\nabla_{\mu}\left(K^{\nu} A_{\nu}\right)=\nabla_{\mu}\left(K^{\nu} A_{\nu}\right), \\
\mathcal{L}_{K} V_{\mu} & =-V_{\mu \nu} K^{\nu}+\nabla_{\mu}\left(V^{\nu} K_{\nu}\right)=0,
\end{aligned}
$$

where $V_{\mu \nu} \equiv \nabla_{\mu} V_{\nu}-\nabla_{\nu} V_{\mu}$. Note that the Killing condition for $V_{\mu}$ is not equivalent to that for $B_{\mu \nu}$. In fact, we do not need the Killing condition for the background field $B_{\mu \nu}$, as we will see in the next section.

\section{$3 \quad$ Ward identities and correlation functions}

In this section, we derive the Ward identities of the $\mathcal{N}=1$ field theory with an $R$-symmetry in $4 \mathrm{D}$ curved space and then comment on some properties of correlation functions, which will be basis of the discussions of the next sections.

\subsection{Ward identities}

The Ward identities corresponding to the symmetries discussed in the previous section can be obtained by using the local renormalization group formalism [23] (see also [24] for a recent review). To this end one first defines the generating functional of connected correlation functions

$$
W\left[g_{\mu \nu}, \psi_{\mu}, A_{\mu}, B_{\mu \nu}\right]=-i \log Z\left[g_{\mu \nu}, \psi_{\mu}, A_{\mu}, B_{\mu \nu}\right],
$$

where $Z\left[g_{\mu \nu}, \psi_{\mu}, A_{\mu}, B_{\mu \nu}\right]$ is the partition function in the presence of the non-dynamical background sources, i.e. $Z=\int[\mathcal{D} \Phi] \exp i S\left[\Phi ; g_{\mu \nu}, \psi_{\mu}, A_{\mu}, B_{\mu \nu}\right]$ ( $\Phi$ represents generic matter fields), and the usual expectation values are defined as $\langle\ldots\rangle \equiv Z^{-1} \int[\mathcal{D} \Phi] \ldots \exp i S$. The gravitino background $\psi_{\mu}$ is set to zero at the end of the computations, since we consider the bosonic backgrounds. Recall that the variation of the generating functional $W$ is given by

$$
\delta W=\int \mathrm{d}^{4} x \boldsymbol{e}\left[-\frac{1}{2}\left\langle\mathcal{T}^{\mu \nu}\right\rangle \delta g_{\mu \nu}+\delta \bar{\psi}_{\mu}\left\langle\mathcal{S}^{\mu}\right\rangle+\left\langle\mathcal{J}^{\mu}\right\rangle \delta G_{\mu}+\frac{1}{4} \varepsilon^{\mu \nu \rho \lambda}\left\langle\mathcal{F}_{\mu \nu}\right\rangle \delta B_{\rho \lambda}\right],
$$

where $G_{\mu} \equiv A_{\mu}-\frac{3}{2} V_{\mu}$. (3.2) gives the definition of the one-point functions of the operators in the presence of arbitrary sources. Namely, (3.2) implies that

$$
\begin{aligned}
\left\langle\mathcal{T}^{\mu \nu}(x)\right\rangle_{g_{\mu \nu}, \psi_{\mu}, A_{\mu}, B_{\mu \nu}} & =-\frac{2}{\boldsymbol{e}} \frac{\delta W}{\delta g_{\mu \nu}(x)}, \\
\left\langle\mathcal{S}^{\mu}(x)\right\rangle_{g_{\mu \nu}, \psi_{\mu}, A_{\mu}, B_{\mu \nu}} & =\frac{1}{e} \frac{\delta W}{\delta \bar{\psi}_{\mu}(x)}, \\
\left\langle\mathcal{J}^{\mu}(x)\right\rangle_{g_{\mu \nu}, \psi_{\mu}, A_{\mu}, B_{\mu \nu}} & =\frac{1}{e} \frac{\delta W}{\delta A_{\mu}(x)}, \\
\left\langle\mathcal{F}_{\mu \nu}(x)\right\rangle_{g_{\mu \nu}, \psi_{\mu}, A_{\mu}, B_{\mu \nu}} & =\frac{1}{e} \varepsilon_{\mu \nu \rho \lambda} \frac{\delta W}{\delta B_{\rho \lambda}(x)} .
\end{aligned}
$$


The Ward identities corresponding to the symmetries (2.3), (2.4) and (2.5) are obtained by requiring $\delta W=0$ up to potential quantum anomalies for the variations (2.3)-(2.5), and the results are as follows:

$$
\begin{aligned}
0= & -\mathcal{D}_{\mu}\left\langle\overline{\mathcal{S}}^{\mu}\right\rangle+\frac{i}{2}\left\langle\overline{\mathcal{S}}^{\mu}\right\rangle \gamma_{\mu}\left(V^{\rho} \gamma_{\rho} \gamma_{*}\right)+\frac{1}{2} \bar{\psi}_{\mu} \gamma_{\nu}\left\langle\mathcal{T}^{\mu \nu}\right\rangle+\frac{3}{8}\left[\left(\bar{\psi}_{\mu} \gamma_{\nu}+\bar{\psi}_{\nu} \gamma_{\mu}\right) V^{\nu}\left\langle\mathcal{J}^{\mu}\right\rangle\right. \\
& \left.-\bar{\psi}_{\rho} \gamma^{\rho} V_{\mu}\left\langle\mathcal{J}^{\mu}\right\rangle-\bar{\psi}_{\lambda} \gamma_{\rho} \varepsilon^{\mu \nu \rho \lambda} \nabla_{\nu}\left\langle\mathcal{J}_{\mu}\right\rangle-i \varepsilon^{\mu \rho \sigma \nu}\left\langle\mathcal{J}_{\sigma}\right\rangle V_{\rho} \bar{\psi}_{\mu} \gamma_{*} \gamma_{\nu}\right] \\
& +\frac{i}{4} \mathcal{D}_{\lambda} \bar{\psi}_{\sigma}\left(-i \varepsilon^{\lambda \sigma}{ }_{\mu \nu} \gamma^{\nu}+\delta_{\mu}^{\sigma} \gamma_{*} \gamma^{\lambda}-\delta_{\mu}^{\lambda} \gamma_{*} \gamma^{\sigma}\right)\left\langle\mathcal{J}^{\mu}\right\rangle+\frac{1}{4} \bar{\psi}_{\mu} \gamma_{\nu} \varepsilon^{\mu \nu \rho \lambda}\left\langle\mathcal{F}_{\rho \lambda}\right\rangle, \\
\mathcal{A}_{\text {diffeo }}= & \nabla^{\mu}\left\langle\mathcal{T}_{\mu \nu}\right\rangle-\left\langle\mathcal{J}^{\mu}\right\rangle G_{\mu \nu}-\nabla_{\mu}\left\langle\mathcal{J}^{\mu}\right\rangle G_{\nu}+\frac{1}{4} \varepsilon^{\mu \kappa \rho \lambda}\left\langle\mathcal{F}_{\mu \kappa}\right\rangle\left(\nabla_{\nu} B_{\rho \lambda}+\nabla_{\rho} B_{\lambda \nu}+\nabla_{\lambda} B_{\nu \rho}\right), \\
\mathcal{A}_{\text {chiral }}= & \nabla_{\mu}\left\langle\mathcal{J}^{\mu}\right\rangle .
\end{aligned}
$$

Here

$$
G_{\mu \nu} \equiv \nabla_{\mu} G_{\nu}-\nabla_{\nu} G_{\mu}, \quad \mathcal{D}_{\nu} \overline{\mathcal{S}}^{\mu} \equiv \nabla_{\nu} \overline{\mathcal{S}}^{\mu}-G_{\nu}\left(i \gamma_{*} \overline{\mathcal{S}}^{\mu}\right)
$$

and the gravitino-dependent terms are omitted in (3.5) and (3.6). In (3.4) it is assumed that there is no supersymmetry anomaly.

The diffeomorphism anomaly $\mathcal{A}_{\text {diffeo }}$ and the $\mathrm{U}(1)_{R}$ chiral anomaly $\mathcal{A}_{\text {chiral }}$ need further explanation. The chiral anomaly is usually accompanied by the mixed gravitational anomaly, which breaks the classical diffeomorphism invariance. The anomalies are given by

$$
\begin{aligned}
& \mathcal{A}_{\text {chiral }}=\frac{1}{4} \varepsilon^{\kappa \sigma \alpha \beta}\left[c_{A} G_{\kappa \sigma} G_{\alpha \beta}+(1-\alpha) c_{m} R_{\lambda \kappa \sigma}^{\nu} R_{\nu \alpha \beta}^{\lambda}\right], \\
& \mathcal{A}_{\text {diffeo }}=-\alpha c_{m} g^{\mu \nu} \frac{1}{\sqrt{-g}} \partial_{\lambda}\left[\sqrt{-g} \frac{1}{2} \varepsilon^{\kappa \sigma \alpha \beta} G_{\kappa \sigma} \partial_{\alpha} \Gamma_{\mu \beta}^{\lambda}\right],
\end{aligned}
$$

see e.g. [25] for a recent review. Here the coefficients $c_{A}$ and $c_{m}$ are determined according to the field content of the theory and are related to the central charges. The scheme parameter $\alpha$ is the coefficient of the diffeomorphism and gauge non-invariant contact counterterm that determines where the mixed anomaly appears: if $\alpha=0$, then the mixed anomaly appears only in the $\mathrm{U}(1)_{R}$ Ward identity, while if $\alpha=1$ it appears only in the diffeomorphism Ward identity. In this note we choose a scheme $\alpha=0$ such that the mixed anomaly does not appear in the diffeomorphism Ward identity.

We emphasize that even if we mainly consider bosonic backgrounds in quantum field theory, we must keep the gravitino background field in (3.4) in order to compute two-point functions of the supercurrent operator. In principle, the Ward identities (3.5) and (3.6) also contain the gravitino-dependent terms, which we ignore since they are irrelevant unless we differentiate (3.5) and (3.6) with respect to the gravitino.

\subsection{Higher-point correlation functions}

Taking further derivatives of (3.3) with respect to the sources gives higher-point correlation functions, for which we use double bra-ket notation $\langle\langle\ldots\rangle\rangle$, i.e.

$$
\left\langle\left\langle\mathcal{O}_{j_{1}}\left(x_{1}\right) \mathcal{O}_{j_{2}}\left(x_{2}\right) \cdots \mathcal{O}_{j_{n}}\left(x_{n}\right)\right\rangle\right\rangle \equiv \frac{\Delta}{\Delta \mathcal{B}_{j_{n}}\left(x_{n}\right)} \ldots \frac{\Delta\left\langle\mathcal{O}_{j_{1}}\left(x_{1}\right)\right\rangle}{\Delta \mathcal{B}_{j_{2}}\left(x_{2}\right)}=\frac{\Delta}{\Delta \mathcal{B}_{j_{n}}\left(x_{n}\right)} \cdots \frac{\Delta}{\Delta \mathcal{B}_{j_{2}}\left(x_{2}\right)} \frac{\Delta(i W)}{\Delta \mathcal{B}_{j_{1}}\left(x_{1}\right)} .
$$


Here $\mathcal{O}_{j}$ stands for any operator in the $\mathcal{R}$-multiplet, i.e. $\mathcal{O}_{j}=\left\{\mathcal{T}^{\mu \nu}, \mathcal{S}_{\alpha}^{\mu}, \mathcal{J}^{\mu}, \mathcal{F}^{\mu \nu}\right\}$, and $\frac{\Delta}{\Delta \mathcal{B}_{j}}$ is a suitably defined functional derivative with respect to the background sources $\mathcal{B}_{j}=\left\{g_{\mu \nu}, \bar{\psi}_{\mu}, A_{\mu}, B_{\rho \lambda}\right\}$ as

$$
\frac{\Delta}{\Delta \mathcal{B}_{j}(x)}=\left\{-\frac{2}{i \boldsymbol{e}} \frac{\delta}{\delta g_{\mu \nu}(x)}, \frac{1}{i \boldsymbol{e}} \frac{\delta}{\delta \bar{\psi}_{\mu}(x)}, \frac{1}{i \boldsymbol{e}} \frac{\delta}{\delta A_{\mu}(x)} \cdot \frac{\varepsilon_{\mu \nu \rho \lambda}}{i \boldsymbol{e}} \frac{\delta}{\delta B_{\rho \lambda}(x)}\right\}
$$

so that $\mathcal{O}_{j}(x)=\frac{\Delta(i S)}{\Delta \mathcal{B}_{j}(x)}$ according to $(2.2)$. For instance,

$$
\begin{aligned}
\left\langle\left\langle\mathcal{T}^{\mu \nu}(x) \mathcal{J}^{\rho}(y)\right\rangle\right\rangle & \equiv \frac{1}{i \boldsymbol{e}} \frac{\delta\left\langle\mathcal{T}^{\mu \nu}(x)\right\rangle}{\delta A_{\rho}(y)}=\frac{1}{i \boldsymbol{e}} \frac{\delta}{\delta A_{\rho}(y)}\left(-\frac{2}{\boldsymbol{e}} \frac{\delta W}{\delta g_{\mu \nu}(x)}\right) \\
\left\langle\left\langle\mathcal{S}^{\mu}(x) \mathcal{J}^{\nu}(y)\right\rangle\right\rangle & \equiv \frac{1}{i \boldsymbol{e}} \frac{\delta\left\langle\mathcal{S}^{\mu}(x)\right\rangle}{\delta A_{\nu}(y)}=\frac{1}{i \boldsymbol{e}} \frac{\delta}{\delta A_{\nu}(y)}\left(\frac{1}{\boldsymbol{e}} \frac{\delta W}{\delta \bar{\psi}_{\mu}(x)}\right) \\
\left\langle\left\langle\mathcal{S}^{\mu}(x) \mathcal{J}^{\nu}(y) \mathcal{J}^{\rho}(z)\right\rangle\right\rangle & \equiv \frac{1}{i \boldsymbol{e}} \frac{\delta\left\langle\left\langle\mathcal{S}^{\mu}(x) \mathcal{J}^{\nu}(y)\right\rangle\right\rangle}{\delta A_{\rho}(z)}=\frac{1}{i \boldsymbol{e}} \frac{\delta}{\delta A_{\rho}(z)}\left(\frac{1}{i \boldsymbol{e}} \frac{\delta}{\delta A_{\nu}(y)}\left(\frac{1}{\boldsymbol{e}} \frac{\delta W}{\delta \bar{\psi}_{\mu}(x)}\right)\right) .
\end{aligned}
$$

Notice that we use different notation $\langle\langle\ldots\rangle\rangle$ than the usual one $\langle\ldots\rangle_{C}$ (the subscript $C$ stands for "connected"), though $\langle\langle\ldots\rangle\rangle$ is clearly a connected correlation function as $W$ is the generating functional for connected correlation functions. The reason of using $\langle\langle\ldots\rangle\rangle$ rather than $\langle\ldots\rangle_{C}$ to denote two- and higher-point functions ${ }^{2}$ is that the quantity $\langle\langle\ldots\rangle\rangle$ can differ from $\langle\ldots\rangle_{C}$ by contact terms when the operators depend on the sources due to the non-linear dependence of the action on the sources. For instance, using the definition of $\langle\langle\ldots\rangle\rangle$ given above it can be easily seen that

$$
\begin{aligned}
\left\langle\left\langle\mathcal{J}^{\mu}(x) \mathcal{J}^{\nu}(y)\right\rangle\right\rangle & =\frac{1}{i \boldsymbol{e}} \frac{\delta\left\langle\mathcal{J}^{\mu}(x)\right\rangle}{\delta A_{\nu}(y)}=\left\langle\left(\frac{1}{\boldsymbol{e}} \frac{\delta S}{\delta A_{\nu}(y)}+\frac{1}{i \boldsymbol{e}} \frac{\delta}{\delta A_{\nu}(y)}\right) \mathcal{J}^{\mu}(x)\right\rangle_{C} \\
& =\left\langle\mathcal{J}^{\mu}(x) \mathcal{J}^{\nu}(y)\right\rangle_{C}-i \delta^{4}(x, y)\left\langle\frac{\partial \mathcal{J}^{\mu}(x)}{\partial A_{\nu}(x)}\right\rangle
\end{aligned}
$$

where $\delta^{4}(x, y)=\delta^{4}(x-y) / \boldsymbol{e}$ is the invariant Dirac delta function. In obtaining the last term in the second line above it is used that the action (and hence operator $\mathcal{J}$ ) does not depend on the derivatives of the background sources which are non-dynamical. The contact term above corresponds to the second functional derivative of action $S$ with respect to $A$, i.e. $\left\langle\frac{\delta}{e \delta A_{\nu}(y)} \frac{\delta S}{e \delta A_{\mu}(x)}\right\rangle$, which is non-vanishing when the action $S$ depends non-linearly on the gauge field $A$. For higher-point functions there can be more contact terms, and in general it is not obvious whether or not the contact terms play any role and can be ignored in the calculations.

In order to investigate the potential consequences of the contact terms, let us consider $(n+1)$-point functions obtained by taking functional derivatives of an one-point function

\footnotetext{
${ }^{2}$ For one-point functions we use the usual bra-ket notation $\langle\cdot\rangle$ since in this case both quantities are identical, i.e. $\langle\langle\mathcal{O}(x)\rangle\rangle=\langle\mathcal{O}(x)\rangle$.
} 
of an operator $\mathcal{O}(x)$ with respect to the background sources (see (3.10)), i.e.

$$
\begin{aligned}
\left\langle\left\langle\mathcal{O}(x) \mathcal{O}_{j_{1}}\left(x_{1}\right) \cdots \mathcal{O}_{j_{n}}\left(x_{n}\right)\right\rangle\right\rangle & =\frac{\Delta}{\Delta \mathcal{B}_{j_{n}}\left(x_{n}\right)} \cdots \frac{\Delta}{\Delta \mathcal{B}_{j_{2}}\left(x_{2}\right)} \frac{\Delta}{\Delta \mathcal{B}_{j_{1}}\left(x_{1}\right)}\langle\mathcal{O}(x)\rangle \\
& =\left\langle\left(\mathcal{O}_{j_{n}}\left(x_{n}\right)+\frac{\Delta}{\Delta \mathcal{B}_{j_{n}}\left(x_{n}\right)}\right) \cdots\left(\mathcal{O}_{j_{1}}\left(x_{1}\right)+\frac{\Delta}{\Delta \mathcal{B}_{j_{1}}\left(x_{1}\right)}\right) \mathcal{O}(x)\right\rangle_{C} \\
& =\left\langle\mathcal{O}_{j_{n}}\left(x_{n}\right) \cdots \mathcal{O}_{j_{1}}\left(x_{1}\right) \mathcal{O}(x)\right\rangle_{C} \text { +contact terms, }
\end{aligned}
$$

where the contact terms contain connected correlation functions with less than $n+1$ operators and are proportional to $\delta$-functions that arise whenever $\frac{\Delta}{\Delta \mathcal{B}}$ acts on operators. The contact terms can be split into two parts according to whether it contains $\delta^{4}\left(x, x_{k}\right)$ or not, namely

$$
\text { contact terms }=\text { terms with } \delta^{4}\left(x, x_{k}\right)+\langle\mathcal{O}(x) \ldots\rangle_{C},
$$

where the first part on the r.h.s. of (3.17) consists of terms that contain $\delta^{4}\left(x, x_{k}\right)$ (and their products), which results from $\frac{\Delta}{\Delta \mathcal{B}_{j_{k}}}$ acting on $\mathcal{O}(x)$, while the rest is collected into the second term, which does not contain the derivatives of $\mathcal{O}(x)$ and hence can be written in the form of $\langle\mathcal{O}(x) \ldots\rangle_{C}$ The second part in (3.17) contains $\delta^{4}\left(x_{k}, x_{l}\right)$ (and their products) with $k \neq l$ and is absent when $n=1$ (i.e. for two-point functions). Splitting in the form (3.17) will be useful in the following discussions.

Now we suppose that $\mathcal{O}(x)$ corresponds to a conserved current $X^{\mu}$, i.e. $\mathcal{O}(x)=X^{\mu}(x)$ with $\nabla_{\mu}\left\langle X^{\mu}(x)\right\rangle=0$. When acting $\nabla_{\mu}=e^{-1} \frac{\partial}{\partial x^{\mu}} \boldsymbol{e}$ on (3.17) and taking integration $\int \mathrm{d}^{4} x \boldsymbol{e}$, the first part in (3.17) does not contribute since it leads to the integration of the total derivative of $\delta^{4}\left(x-x_{j}\right)$ that vanishes. In the case of two-point functions, the second part in (3.17) does not exist. Therefore, for the two-point functions all contact terms drop out through the operations mentioned above, so that we have

$$
\int \mathrm{d}^{4} x \boldsymbol{e} \nabla_{\mu}\left\langle\left\langle X^{\mu}(x) \mathcal{O}_{j_{1}}(y)\right\rangle\right\rangle=\int \mathrm{d}^{4} x \boldsymbol{e} \nabla_{\mu}\left\langle X^{\mu}(x) \mathcal{O}_{j_{1}}(y)\right\rangle_{C}=\left\langle\left[Q_{X}, \mathcal{O}_{j_{1}}(y)\right]\right\rangle,
$$

where $Q_{X}$ is the corresponding conserved charge defined as $Q_{X} \equiv \int_{\mathcal{C}} \mathrm{d} \sigma_{\mu} X^{\mu}$ with $\mathcal{C}$ being the Cauchy surface. ${ }^{3}$ (3.18) will be often used in section 4 . For three-point and higherpoint functions the second part of the contact terms in (3.17) can contribute and needs a careful treatment.

Let us consider the case when $Q_{X}$ annihilates the vacuum state $|\Omega\rangle$, i.e. $Q_{X}|\Omega\rangle=0$. In this case it follows that

$$
Q_{X}|\Omega\rangle=0 \Longrightarrow \int \mathrm{d}^{4} x \boldsymbol{e} \nabla_{\mu}\left\langle\left\langle X^{\mu}(x) \mathcal{O}_{j_{1}}\left(x_{1}\right) \cdots \mathcal{O}_{j_{n}}\left(x_{n}\right)\right\rangle\right\rangle=0
$$

This is analogous to the usual formula $\int \mathrm{d}^{4} x e \nabla_{\mu}\left\langle X^{\mu}(x) \ldots\right\rangle=0$ when $Q_{X}|\Omega\rangle=0$. (3.19) can be shown as follows. First, note that $Q_{X}|\Omega\rangle=0$ leads to $\int \mathrm{d}^{4} x \boldsymbol{e} \nabla_{\mu}\left\langle X^{\mu}(x) \ldots\right\rangle_{C}=0$,

\footnotetext{
${ }^{3}$ One comment is in order on the second equality of (3.18). The usual relation is $\left\langle\left[Q_{X}, \mathcal{O}_{j}(y)\right]\right\rangle=$ $\int \mathrm{d}^{4} x \boldsymbol{e} \nabla_{\mu}\left\langle X^{\mu}(x) \mathcal{O}_{j}(y)\right\rangle$ without subscript $C$. However, since $\nabla_{\mu}\left\langle X^{\mu}(x)\right\rangle=0$, the disconnected connected part vanishes, so that $\left\langle\left[Q_{X}, \mathcal{O}_{j}(y)\right]\right\rangle=\int \mathrm{d}^{4} x e \nabla_{\mu}\left\langle X^{\mu}(x) \mathcal{O}_{j}(y)\right\rangle_{C}$.
} 
which follows from $\int \mathrm{d}^{4} x \boldsymbol{e} \nabla_{\mu}\left\langle X^{\mu}(x) \ldots\right\rangle=0$ and the definition of the connected correlation functions. ${ }^{4}$ Then, the first term (non-contact part) in (3.16) (with $\mathcal{O}(x)=X^{\mu}(x)$ ) and the second part of the contact terms in (3.17) do not contribute to $\int \mathrm{d}^{4} x \boldsymbol{e} \nabla_{\mu}\left\langle\left\langle X^{\mu}(x) \mathcal{O}_{j_{1}}\left(x_{1}\right)\right.\right.$ $\left.\left.\cdots \mathcal{O}_{j_{n}}\left(x_{n}\right)\right\rangle\right\rangle$. Since the first part of the contact terms in (3.17) does not contribute either as mentioned above, we end up with (3.19), which will be employed in section 5 .

\section{Rigid supersymmetry algebra}

Now we recover the rigid supersymmetry algebra on the curved backgrounds by deriving the transformation laws of the supercurrent and $\mathrm{U}(1)_{R}$ current with respect to the rigid supersymmetry. ${ }^{5}$

In order to set up the general strategy, we first deal with the diffeomorphism Ward identity. We multiply (3.5) by an arbitrary vector field $\xi^{\nu}(x)$ and take a functional derivative $\frac{\delta}{i e \delta A_{\rho}(y)}$ to obtain

$$
\begin{aligned}
0= & i \xi^{\nu}\left[\nabla^{\mu}\left\langle\left\langle\mathcal{T}_{\mu \nu}(x) \mathcal{J}^{\rho}(y)\right\rangle\right\rangle-G_{\mu \nu}\left\langle\left\langle\mathcal{J}^{\mu}(x) \mathcal{J}^{\rho}(y)\right\rangle\right\rangle-G_{\nu} \nabla_{\mu}\left\langle\left\langle\mathcal{J}^{\mu}(x) \mathcal{J}^{\rho}(y)\right\rangle\right\rangle\right. \\
& \left.+\frac{1}{4} \varepsilon^{\mu \kappa \rho \lambda}\left(\nabla_{\nu} B_{\rho \lambda}+\nabla_{\rho} B_{\lambda \nu}+\nabla_{\lambda} B_{\nu \rho}\right)\left\langle\left\langle\mathcal{F}_{\mu \kappa}(x) \mathcal{J}^{\rho}(y)\right\rangle\right\rangle\right] \\
& -\delta^{4}(x, y)\left[\nabla_{\nu}\left(\xi^{\nu}\left\langle\mathcal{J}^{\rho}\right\rangle\right)-\left\langle\mathcal{J}^{\nu}\right\rangle \nabla_{\nu} \xi^{\rho}\right]+e^{-1} \partial_{\nu}\left[\delta^{4}(x-y)\left(\xi^{\nu}\left\langle\mathcal{J}^{\rho}\right\rangle-\xi^{\rho}\left\langle\mathcal{J}^{\nu}\right\rangle\right)\right] .
\end{aligned}
$$

The integration over $x$-space (i.e. $\int \mathrm{d}^{4} x \boldsymbol{e}$ ) of the above gives

$$
\begin{aligned}
\nabla_{\nu}\left(\xi^{\nu}\left\langle\mathcal{J}^{\rho}\right\rangle\right)-\left\langle\mathcal{J}^{\nu}\right\rangle \nabla_{\nu} \xi^{\rho}= & \int \mathrm{d}^{4} x \text { e } i \xi^{\nu}\left[\nabla^{\mu}\left\langle\left\langle\mathcal{T}_{\mu \nu}(x) \mathcal{J}^{\rho}(y)\right\rangle\right\rangle-G_{\mu \nu}\left\langle\left\langle\mathcal{J}^{\mu}(x) \mathcal{J}^{\rho}(y)\right\rangle\right\rangle-\right. \\
& \left.-G_{\nu} \nabla_{\mu}\left\langle\left\langle\mathcal{J}^{\mu}(x) \mathcal{J}^{\rho}(y)\right\rangle\right\rangle+\frac{1}{4} \varepsilon^{\mu \kappa \rho \lambda}\left(\nabla_{\nu} B_{\rho \lambda}+\nabla_{\rho} B_{\lambda \nu}+\nabla_{\lambda} B_{\nu \rho}\right)\left\langle\left\langle\mathcal{F}_{\mu \kappa}(x) \mathcal{J}^{\rho}(y)\right\rangle\right\rangle\right],
\end{aligned}
$$

where the left-hand side actually corresponds to the variation of the operator $\mathcal{J}^{\rho}$ under the diffeomorphism associated with the vector $\xi^{\mu}$, see e.g. section 5.2.3 in [26]. It then follows from (4.2) that the $\mathrm{U}(1)_{R}$ current $\mathcal{J}^{\mu}$ transforms as a vector density under the diffeomorphism, which has to do with the fact that the quantity conjugate to the vector source is not a vector but a vector density operator (see e.g. [27]).

\footnotetext{
${ }^{4}$ As an illustration of the statement that $\int \mathrm{d}^{4} x e \nabla_{\mu}\left\langle X^{\mu}(x) \ldots\right\rangle_{C}=0$ if $\nabla_{\mu}\left\langle X^{\mu}(x) \ldots\right\rangle=0$, let us consider connected three-point function $\int \mathrm{d}^{4} x e \nabla_{\mu}\left\langle X^{\mu}(x) \mathcal{O}_{j_{1}}\left(x_{1}\right) \mathcal{O}_{j_{2}}\left(x_{2}\right)\right\rangle_{C}$. From the definition of the connected correlation function we have $\int \mathrm{d}^{4} x e \nabla_{\mu}\left\langle X^{\mu}(x) \mathcal{O}_{j_{1}}\left(x_{1}\right) \mathcal{O}_{j_{2}}\left(x_{2}\right)\right\rangle_{C}=\int \mathrm{d}^{4} x e \nabla_{\mu}\left\langle X^{\mu}(x) \mathcal{O}_{j_{1}}\left(x_{1}\right)\right.$ $\left.\mathcal{O}_{j_{2}}\left(x_{2}\right)\right\rangle-\int \mathrm{d}^{4} x \boldsymbol{e} \nabla_{\mu}\left\langle X^{\mu}(x)\right\rangle\left\langle\mathcal{O}_{j_{1}}\left(x_{1}\right) \mathcal{O}_{j_{2}}\left(x_{2}\right)\right\rangle-\left\langle\mathcal{O}_{j_{1}}\left(x_{1}\right)\right\rangle \int \mathrm{d}^{4} x \boldsymbol{e} \nabla_{\mu}\left\langle X^{\mu}(x) \mathcal{O}_{j_{2}}\left(x_{2}\right)\right\rangle-\int \mathrm{d}^{4} x \boldsymbol{e} \nabla_{\mu}\left\langle X^{\mu}(x)\right.$ $\left.\mathcal{O}_{j_{1}}\left(x_{1}\right)\right\rangle\left\langle\mathcal{O}_{j_{2}}\left(x_{1}\right)\right\rangle+2 \int \mathrm{d}^{4} x \boldsymbol{e} \nabla_{\mu}\left\langle X^{\mu}(x)\right\rangle\left\langle\mathcal{O}_{j_{1}}\left(x_{1}\right)\right\rangle\left\langle\mathcal{O}_{j_{2}}\left(x_{2}\right)\right\rangle$, each term of which contains $\int \mathrm{d}^{4} x \boldsymbol{e} \nabla_{\mu}$ $\left\langle X^{\mu}(x) \ldots\right\rangle=0$, so we have $\int \mathrm{d}^{4} x e \nabla_{\mu}\left\langle X^{\mu}(x) \mathcal{O}_{j_{1}}\left(x_{1}\right) \mathcal{O}_{j_{2}}\left(x_{2}\right)\right\rangle_{C}=0$. This fact is easily generalized to the arbitrary higher-point functions.

${ }^{5}$ We suspect that these transformation rules should be known, but we found only their flat-space version in the literature, see e.g. [10, 12].
} 
Multiplying (3.5) by $K^{\nu}$ defined in (2.8) and assuming that $K^{\mu} A_{\mu}$ is made constant ${ }^{6}$ by a suitable $\mathrm{U}(1)_{R}$ gauge transformation, we obtain ${ }^{7}$

$$
\nabla_{\mu}\left\langle\mathcal{C}_{K}^{\mu}(x)\right\rangle=0
$$

where

$$
\mathcal{C}_{K}^{\mu}(x) \equiv K_{\nu}\left[\mathcal{T}^{\mu \nu}-\mathcal{J}^{\mu}\left(A^{\nu}-\frac{3}{2} V^{\nu}\right)+\frac{1}{2} \varepsilon^{\mu \nu \rho \lambda} \mathcal{F}_{\rho \lambda}\right](x)
$$

This allows us to define a conserved charge

$$
Q_{K} \equiv \int_{\mathcal{C}} \mathrm{d} \sigma_{\mu} \mathcal{C}_{K}^{\mu}
$$

where $\mathcal{C}$ is any Cauchy surface. Now using (3.18) and replacing $\xi$ by $K$ in (4.2), we obtain

$$
i\left\langle\left[Q_{K}, \mathcal{J}^{\rho}\right]\right\rangle=\int \mathrm{d}^{4} x \text { e } i \nabla_{\mu}\left\langle\left\langle\mathcal{C}_{K}^{\mu}(x) \mathcal{J}^{\rho}(y)\right\rangle\right\rangle=\nabla_{\nu}\left(K^{\nu}\left\langle\mathcal{J}^{\rho}\right\rangle\right)-\left\langle\mathcal{J}^{\nu}\right\rangle \nabla_{\nu} K^{\rho} .
$$

Now we use the above strategy to recover the rigid supersymmetry algebra for $\mathcal{N}=1$ field theories with an $R$-symmetry. Multiplying (3.4) by the GKS $\zeta(x)$ and setting the gravitino to zero gives

$$
\nabla_{\mu}\left(\left\langle\overline{\mathcal{S}}^{\mu} \zeta(x)\right\rangle\right)=0
$$

which allows us to define a conserved supercharge associated with the GKS $\zeta$ as

$$
\mathcal{Q}_{\zeta} \equiv \int_{\mathcal{C}} \mathrm{d} \sigma_{\mu} \overline{\mathcal{S}}^{\mu} \zeta
$$

Independence of $\mathcal{Q}_{\zeta}$ on the choice for the Cauchy surface, i.e. conservation of $\mathcal{Q}_{\zeta}$ is an immediate consequence of the Ward identity (3.4) on the bosonic background. Note that we can also define a conserved supercharge $\mathcal{Q}_{\eta}$ associated with the GKS $\eta=i \gamma_{*} \zeta$. Now we multiply (3.4) by the GKS $\zeta$ and taking the functional derivative $\frac{1}{i \boldsymbol{e}} \frac{\delta}{\delta \psi_{\mu}(y)}$ to obtain

$$
\begin{aligned}
i \nabla_{\nu}\left\langle\left\langle\left(\overline{\mathcal{S}}^{\nu} \zeta\right)(x) \mathcal{S}^{\mu}(y)\right\rangle\right\rangle= & \delta^{4}(x, y)\left[\frac { 1 } { 4 } \gamma _ { \nu } \zeta \left(2\left\langle\mathcal{T}^{\mu \nu}\right\rangle+3\left\langle\mathcal{J}^{\mu}\right\rangle V^{\nu}+\left\langle\mathcal{J}^{\nu}\right\rangle V^{\mu}-g^{\mu \nu}\left\langle\mathcal{J}^{\rho}\right\rangle V_{\rho}+\varepsilon^{\mu \nu \rho \lambda}\left\langle\mathcal{F}_{\rho \lambda}\right\rangle\right.\right. \\
& \left.\left.-\frac{1}{2} \varepsilon^{\mu \nu \lambda \sigma} \nabla_{\lambda}\left\langle\mathcal{J}_{\sigma}\right\rangle\right)+\frac{i}{4} \gamma_{*} \gamma_{\nu} \zeta\left(g^{\mu \nu} \nabla_{\rho}\left\langle\mathcal{J}^{\rho}\right\rangle-\nabla^{\nu}\left\langle\mathcal{J}^{\mu}\right\rangle+\varepsilon^{\mu \nu \sigma \kappa}\left\langle\mathcal{J}_{\sigma}\right\rangle V_{\kappa}\right)\right] \\
& +\frac{i}{4} e^{-1} \partial_{\lambda}\left[\delta^{4}(x-y)\left(-i \varepsilon^{\lambda \mu}{ }_{\sigma \nu} \gamma^{\nu}+\delta_{\sigma}^{\mu} \gamma_{*} \gamma^{\lambda}-\delta_{\sigma}^{\lambda} \gamma_{*} \gamma^{\mu}\right) \zeta\left\langle\mathcal{J}^{\sigma}\right\rangle\right]
\end{aligned}
$$

\footnotetext{
${ }^{6}$ When $K^{\mu} A_{\mu}$ is not constant, it seems that there are several inconsistencies in constructing the supersymmetry algebra. For instance, one can not define a conserved charge associated with the Killing vector $K$, and the Lie derivative of $\zeta$ with respect to $K$ does not satisfy the generalized Killing condition.

${ }^{7} \mathrm{~A}$ careful derivation is necessary for the term containing $\mathcal{F}_{\rho \lambda}$. First of all, we have $\nabla_{\mu}\left(\varepsilon^{\mu \nu \rho \lambda} K_{\nu} \mathcal{F}_{\rho \lambda}\right)=$ $4 V^{\mu} K^{\nu} \mathcal{F}_{\mu \nu}=\varepsilon^{\mu \rho \sigma \lambda} K^{\nu}\left(\nabla_{\rho} B_{\sigma \lambda}\right) \mathcal{F}_{\mu \nu}$. Combining this with $\varepsilon^{[\mu \rho \sigma \lambda} K^{\nu]}\left(\nabla_{\rho} B_{\sigma \lambda}\right) \mathcal{F}_{\mu \nu}=0$, which implies $\varepsilon^{\mu \rho \sigma \lambda} K^{\nu}\left(\nabla_{\rho} B_{\sigma \lambda}\right) \mathcal{F}_{\mu \nu}=\frac{1}{2} \mathcal{F}_{\mu \nu} \varepsilon^{\sigma \lambda \mu \nu} K^{\rho}\left(\nabla_{\rho} B_{\sigma \lambda}+\nabla_{\lambda} B_{\rho \sigma}+\nabla_{\sigma} B_{\lambda \rho}\right)$, gives $\nabla_{\mu}\left(\varepsilon^{\mu \nu \rho \lambda} K_{\nu} \mathcal{F}_{\rho \lambda}\right)=$ $\frac{1}{2} \mathcal{F}_{\mu \nu} \varepsilon^{\sigma \lambda \mu \nu} K^{\rho}\left(\nabla_{\rho} B_{\sigma \lambda}+\nabla_{\lambda} B_{\rho \sigma}+\nabla_{\sigma} B_{\lambda \rho}\right)$. Notice that we do not need the Killing condition for $B_{\mu \nu}$.
} 
where we have set the gravitino background to zero at the end. Integrating (4.9) over $x$-space and using (3.18) give

$$
\begin{aligned}
i\left\langle\left[\mathcal{Q}_{\zeta}, \mathcal{S}^{\mu}\right]\right\rangle= & \int \mathrm{d}^{4} x \boldsymbol{e} i \nabla_{\nu}\left\langle\left\langle\left(\overline{\mathcal{S}}^{\nu} \zeta\right)(x) \mathcal{S}^{\mu}(y)\right\rangle\right\rangle \\
= & \frac{1}{4} \gamma_{\nu} \zeta\left(2\left\langle\mathcal{T}^{\mu \nu}\right\rangle+3\left\langle\mathcal{J}^{\mu}\right\rangle V^{\nu}+\left\langle\mathcal{J}^{\nu}\right\rangle V^{\mu}-g^{\mu \nu}\left\langle\mathcal{J}^{\rho}\right\rangle V_{\rho}+\varepsilon^{\mu \nu \rho \lambda}\left\langle\mathcal{F}_{\rho \lambda}\right\rangle-\frac{1}{2} \varepsilon^{\mu \nu \lambda \sigma} \nabla_{\lambda}\left\langle\mathcal{J}_{\sigma}\right\rangle\right)+ \\
& +\frac{i}{4} \gamma_{*} \gamma_{\nu} \zeta\left(g^{\mu \nu} \nabla_{\rho}\left\langle\mathcal{J}^{\rho}\right\rangle-\nabla^{\nu}\left\langle\mathcal{J}^{\mu}\right\rangle+\varepsilon^{\mu \nu \sigma \kappa}\left\langle\mathcal{J}_{\sigma}\right\rangle V_{\kappa}\right) .
\end{aligned}
$$

Note that using (4.10) we can rewrite (4.9) in a simple form as

$$
\nabla_{\nu}\left\langle\left\langle\left(\overline{\mathcal{S}}^{\nu} \zeta\right)(x) \mathcal{S}^{\mu}(y)\right\rangle\right\rangle=\delta^{4}(x, y)\left\langle\left[\mathcal{Q}_{\zeta}, \mathcal{S}^{\mu}\right]\right\rangle+\frac{1}{4} e^{-1} \partial_{\lambda}\left[\delta^{4}(x-y)\left(-i \varepsilon^{\lambda \mu}{ }_{\sigma \nu} \gamma^{\nu}+\delta_{\sigma}^{\mu} \gamma_{*} \gamma^{\lambda}-\delta_{\sigma}^{\lambda} \gamma_{*} \gamma^{\mu}\right) \zeta\left\langle\mathcal{J}^{\sigma}\right\rangle\right] .
$$

Multiplying (4.10) by $\bar{\zeta}$ and $\bar{\eta}$ gives respectively (omitting the bra-ket notation $\langle\cdot\rangle$ )

$$
\begin{aligned}
i\left[\mathcal{Q}_{\zeta}, \bar{\zeta} \mathcal{S}^{\mu}\right] & =\frac{1}{4} \nabla_{\rho}\left(K^{\mu} \mathcal{J}^{\rho}-K^{\rho} \mathcal{J}^{\mu}\right), \\
i\left[\mathcal{Q}_{\zeta}, \bar{\eta} \mathcal{S}^{\mu}\right] & =\frac{1}{2} K_{\nu}\left[\mathcal{T}^{\mu \nu}-G^{\nu} \mathcal{J}^{\mu}+\frac{1}{2} \varepsilon^{\mu \nu \rho \lambda} \mathcal{F}_{\rho \lambda}\right]-\frac{1}{8} \nabla_{\lambda}\left(\varepsilon^{\mu \nu \lambda \sigma} K_{\nu} \mathcal{J}_{\sigma}\right)+\frac{1}{2}\left(K^{\nu} A_{\nu}\right) \mathcal{J}^{\mu} .
\end{aligned}
$$

It then follows that

$$
\begin{aligned}
\nabla_{\mu}\left[\mathcal{Q}_{\zeta}, \bar{\zeta} \mathcal{S}^{\mu}\right] & =0, \quad\left[\mathcal{Q}_{\zeta}, \mathcal{Q}_{\zeta}\right]=0 \\
i\left[\mathcal{Q}_{\zeta}, \mathcal{Q}_{\eta}\right] & =\frac{1}{2} Q_{K}+\frac{1}{2}\left(K^{\nu} A_{\nu}\right) Q_{R}
\end{aligned}
$$

where $Q_{R}$ is the $\mathrm{U}(1)_{R}$ charge.

Now we multiply (3.4) by the GKS $\zeta(x)$, differentiate it with respect to $A_{\mu}(y)$ and $B_{\sigma \lambda}(y)$, respectively (cf. (3.3)), and set the gravitino to zero. Then, we get

$$
\nabla_{\nu}\left\langle\left\langle\left(\overline{\mathcal{S}}^{\nu} \zeta\right) \mathcal{J}^{\mu}\right\rangle\right\rangle=\left\langle\overline{\mathcal{S}}^{\mu}\right\rangle \gamma_{*} \zeta \delta^{4}(x, y), \quad \nabla_{\rho}\left\langle\left\langle\left(\overline{\mathcal{S}}^{\rho} \zeta\right) \mathcal{F}_{\mu \nu}\right\rangle\right\rangle=\frac{1}{2}\left[\nabla_{\mu}\left(\left\langle\overline{\mathcal{S}}^{\rho}\right\rangle \gamma_{\rho} \gamma_{\nu} \gamma_{*} \zeta\right)-(\mu \leftrightarrow \nu)\right] \delta^{4}(x, y),
$$

which lead to

$$
\begin{aligned}
{\left[\mathcal{Q}_{\zeta}, \mathcal{J}^{\mu}\right] } & =\overline{\mathcal{S}}^{\mu} \gamma_{*} \zeta, \quad i\left[\mathcal{Q}_{\zeta}, Q_{R}\right]=\mathcal{Q}_{\eta}, \\
{\left[\mathcal{Q}_{\zeta}, \mathcal{F}_{\mu \nu}\right] } & =\frac{1}{2}\left[\nabla_{\mu}\left(\overline{\mathcal{S}}^{\rho} \gamma_{\rho} \gamma_{\nu} \gamma_{*} \zeta\right)-(\mu \leftrightarrow \nu)\right] .
\end{aligned}
$$

These transformation laws of the currents $\mathcal{S}^{\mu}, \mathcal{J}^{\mu}$ and $\mathcal{F}_{\mu \nu}$ under the rigid supersymmetry are explicitly checked in appendix B for a free chiral theory on $\mathbb{R} \times S^{3}$.

Transformation law for the supercharge $\mathcal{Q}_{\zeta}$ under the diffeomorphism associated with the Killing vector $K$ can be obtained by differentiating the diffeomorphism Ward identity with respect to the gravitino source. For this, the diffeomorphism Ward identity should 
be extended to involve the gravitino-dependent terms. We do not present the details of its calculation here, but give the final result as

$$
i\left[Q_{K}, \mathcal{Q}_{\zeta}\right]=-\left(K^{\mu} A_{\mu}\right) \mathcal{Q}_{\eta} \quad \text { or } \quad\left[Q_{K}+\left(K^{\mu} A_{\mu}\right) Q_{R}, \mathcal{Q}_{\zeta}\right]=0
$$

which is consistent with $(2.9 \mathrm{~b})$.

In summary, the supersymmetry algebra is

$$
\begin{aligned}
i\left[\mathcal{Q}_{\zeta}, \mathcal{Q}_{\eta}\right] & =\frac{1}{2} Q_{K}+\frac{1}{2}\left(K^{\nu} A_{\nu}\right) Q_{R}, \\
{\left[Q_{K}+\left(K^{\mu} A_{\mu}\right) Q_{R}, \mathcal{Q}_{\zeta}\right] } & =0, \quad i\left[\mathcal{Q}_{\zeta}, Q_{R}\right]=\mathcal{Q}_{\eta}, \\
{\left[Q_{K}+\left(K^{\mu} A_{\mu}\right) Q_{R}, \mathcal{Q}_{\zeta}\right] } & =0
\end{aligned}
$$

see e.g. [7].

We end this section by addressing the quantum consistency of the $\mathcal{N}=1$ rigid supersymmetry algebra (4.20). First, note that by differentiating (3.6) with respect to the source field $A_{\nu}(y)$, we get

$$
i \nabla_{\mu}\left\langle\left\langle\mathcal{J}^{\mu}(x) \mathcal{J}^{\nu}(y)\right\rangle\right\rangle=c_{A} \varepsilon^{\mu \nu \rho \sigma} \nabla_{\mu} \delta^{4}(x, y) G_{\rho \sigma},
$$

where the right-hand side is a total derivative. It then follows that

$$
\int \mathrm{d}^{4} x \boldsymbol{e} \nabla_{\mu}\left\langle\left\langle\mathcal{J}^{\mu}(x) \mathcal{J}^{\nu}(y)\right\rangle\right\rangle=0
$$

which implies that $\left[Q_{R}, \mathcal{J}^{\nu}\right]=0$. Using this, we find from (4.20a) and (4.6) that

$$
2\left[\left[\mathcal{Q}_{\zeta}, \mathcal{Q}_{\eta}\right], \mathcal{J}^{\mu}\right]=-i\left[Q_{K}, \mathcal{J}^{\mu}\right]=-\nabla_{\nu}\left(K^{\nu} \mathcal{J}^{\mu}\right)+\mathcal{J}^{\nu} \nabla_{\nu} K^{\mu}
$$

On the other hand, (4.17) and (4.12) imply that

$$
2\left[\mathcal{Q}_{\zeta},\left[\mathcal{Q}_{\eta}, \mathcal{J}^{\mu}\right]\right]=2 i\left[\mathcal{Q}_{\zeta}, \bar{\zeta} \mathcal{S}^{\mu}\right]=\frac{1}{2} \nabla_{\nu}\left(K^{\mu} \mathcal{J}^{\nu}-K^{\nu} \mathcal{J}^{\mu}\right)
$$

and therefore

$$
2\left[\left[\mathcal{Q}_{\zeta}, \mathcal{Q}_{\eta}\right], \mathcal{J}^{\mu}\right]=2\left[\mathcal{Q}_{\zeta},\left[\mathcal{Q}_{\eta}, \mathcal{J}^{\mu}\right]\right]-2\left[\mathcal{Q}_{\eta},\left[\mathcal{Q}_{\zeta}, \mathcal{J}^{\mu}\right]\right]=\nabla_{\nu}\left(K^{\mu} \mathcal{J}^{\nu}-K^{\nu} \mathcal{J}^{\mu}\right)
$$

Since $K$ is a nowhere vanishing vector [7], (4.23) can be consistent with (4.25) only when

$$
\nabla_{\mu}\left\langle\mathcal{J}^{\mu}\right\rangle=0
$$

or equivalently (see (3.6) and (3.8))

$$
\varepsilon^{\mu \nu \rho \sigma} G_{\mu \nu} G_{\rho \sigma}=0, \quad \varepsilon^{\kappa \sigma \alpha \beta} R_{\lambda \kappa \sigma}^{\nu} R_{\nu \alpha \beta}^{\lambda}=0,
$$

if we assume $c_{A} \neq 0$ and $c_{m} \neq 0$. These are additional constraints imposed on the background sources since the GKS condition (2.7) does not automatically imply (4.27) [15]. Does the condition (4.27) suffice for consistent construction of $\mathcal{N}=1$ field theory with an $R$-symmetry when $c_{A} \neq 0$ and $c_{m} \neq 0$ ? As we will see in the next section the answer is no, due to a problem that manifests itself in the higher-point correlation functions. 


\section{Quantum consistency}

In this section we show that the $\mathrm{U}(1)_{R}$ coefficient $c_{A}$ should vanish in order for the newminimal supergravity formulation of the $\mathcal{N}=1$ field theory with an $R$-symmetry to be quantum-mechanically consistent.

Condition (4.27) is related to the $\mathcal{N}=1$ rigid supersymmetry algebra (4.20), which relies on the assumption that $K^{\mu} A_{\mu}$ is constant. Now we would like to pursue our investigation without using this assumption. Instead, following [16] we suppose that the vacuum state $|\Omega\rangle$ is supersymmetric, i.e.

$$
\mathcal{Q}_{\zeta}|\Omega\rangle=\mathcal{Q}_{\eta}|\Omega\rangle=0
$$

which implies $\left\langle\delta_{\zeta}(\ldots)\right\rangle=\left\langle\delta_{\eta}(\ldots)\right\rangle=0$ with $\delta_{\zeta}(\ldots) \equiv\left[\mathcal{Q}_{\zeta}, \ldots\right]$ and $\delta_{\eta}(\ldots) \equiv\left[\mathcal{Q}_{\eta}, \ldots\right]$. Notice that when $K^{\mu} A_{\mu}$ is not constant, the right-hand side of (4.3) becomes non-zero, i.e. $\nabla_{\mu} \mathcal{C}^{\mu}=-\mathcal{J}^{\mu} \nabla_{\mu}\left(A_{\nu} K^{\nu}\right)$, and (4.13) therefore leads to

$$
0=i \nabla_{\mu}\left\langle\left[\mathcal{Q}_{\zeta}, \bar{\eta} \mathcal{S}^{\mu}\right]\right\rangle=\frac{1}{2} K^{\nu} A_{\nu} \nabla_{\mu}\left\langle\mathcal{J}^{\mu}\right\rangle=\frac{1}{2} K^{\nu} A_{\nu} \mathcal{A}_{\text {chiral }}
$$

on the supersymmetric vacuum. This implies that

$$
K^{\nu} A_{\nu}=0 \quad \text { or } \quad \nabla_{\mu}\left\langle\mathcal{J}^{\mu}\right\rangle=\mathcal{A}_{\text {chiral }}=0 .
$$

Yet this does not seem to cause a serious problem, as we saw the similar constraints in the previous section, see (4.26)-(4.27). However, it turns out that the real problem shows up in the higher-point functions. To see this, we multiply (3.4) by any spinor $\epsilon(x)$ and differentiate with respect to $\bar{\psi}_{\mu}\left(x_{1}\right)$ and then $A_{\nu}\left(x_{2}\right)$. We then set the gravitino to zero and obtain

$$
\begin{aligned}
i \mathcal{D}_{\rho} & \left\langle\left\langle\overline{\mathcal{S}}^{\rho} \epsilon(x) \mathcal{S}^{\mu}\left(x_{1}\right) \mathcal{J}^{\nu}\left(x_{2}\right)\right\rangle\right\rangle+\frac{1}{2}\left\langle\left\langle\overline{\mathcal{S}}^{\lambda} \gamma_{\lambda} V^{\rho} \gamma_{\rho} \gamma_{*} \epsilon(x) \mathcal{S}^{\mu}\left(x_{1}\right) \mathcal{J}^{\nu}\left(x_{2}\right)\right\rangle\right\rangle= \\
= & \frac{1}{2} \delta^{4}\left(x_{1}, x\right) \gamma_{\lambda} \epsilon\left\langle\left\langle\left[\mathcal{T}^{\mu \lambda}+\frac{3}{4}\left(V^{\lambda} \mathcal{J}^{\mu}+V^{\mu} \mathcal{J}^{\lambda}-g^{\mu \lambda} V_{\rho} \mathcal{J}^{\rho}-\varepsilon^{\mu \lambda \rho \kappa} \nabla_{\rho} \mathcal{J}_{\kappa}\right)+\frac{1}{2} \varepsilon^{\mu \lambda \rho \kappa} \mathcal{F}_{\rho \kappa}\right]\left(x_{1}\right) \mathcal{J}^{\nu}\left(x_{2}\right)\right\rangle\right\rangle \\
& +\frac{3 i}{8} \delta^{4}\left(x_{1}, x\right) \gamma_{*} \gamma_{\lambda} \epsilon \varepsilon^{\mu \lambda \rho \sigma} V_{\sigma}\left\langle\left\langle\mathcal{J}_{\rho}\left(x_{1}\right) \mathcal{J}^{\nu}\left(x_{2}\right)\right\rangle\right\rangle \\
& -\frac{i}{4} \delta^{4}\left(x_{1}, x\right)\left(-i \varepsilon^{\lambda \mu}{ }_{\sigma \kappa} \gamma^{\kappa}+\delta_{\sigma}^{\mu} \gamma_{*} \gamma^{\lambda}-\delta_{\sigma}^{\lambda} \gamma_{*} \gamma^{\mu}\right) \mathcal{D}_{\lambda}\left(\epsilon\left\langle\left\langle\mathcal{J}^{\sigma}\left(x_{1}\right) \mathcal{J}^{\nu}\left(x_{2}\right)\right\rangle\right\rangle\right) \\
& +\frac{i}{4} e^{-1} \partial_{\lambda}\left[\delta^{4}\left(x_{1}-x\right)\left(-i \varepsilon_{\sigma \kappa}^{\lambda \mu}{ }_{\sigma \kappa} \gamma^{\kappa}+\delta_{\sigma}^{\mu} \gamma_{*} \gamma^{\lambda}-\delta_{\sigma}^{\lambda} \gamma_{*} \gamma^{\mu}\right) \epsilon\left\langle\left\langle\mathcal{J}^{\sigma}\left(x_{1}\right) \mathcal{J}^{\nu}\left(x_{2}\right)\right\rangle\right\rangle\right] \\
& +\frac{1}{4} \delta^{4}\left(x_{1}, x\right) \delta^{4}\left(x_{1}, x_{2}\right)\left(-i \varepsilon^{\nu \mu}{ }_{\sigma \kappa} \gamma^{\kappa}+\delta_{\sigma}^{\mu} \gamma_{*} \gamma^{\nu}-\delta_{\sigma}^{\nu} \gamma_{*} \gamma^{\mu}\right) \gamma_{*} \epsilon\left\langle\mathcal{J}^{\sigma}\right\rangle+i \delta^{4}\left(x_{2}, x\right)\left\langle\left\langle\overline{\mathcal{S}}^{\nu} \gamma_{*} \epsilon(x) \mathcal{S}^{\mu}\left(x_{1}\right)\right\rangle\right\rangle .
\end{aligned}
$$

As mentioned before, it is important to keep all contact terms in the above computation. Now we let $\epsilon(x)$ be the GKS $\zeta$ and multiply (5.4) by $\bar{\eta}=i \bar{\zeta}\left(x_{1}\right) \gamma_{*}$. We then obtain

$$
\begin{aligned}
i \nabla_{\rho} & \left\langle\left\langle\overline{\mathcal{S}}^{\rho} \zeta(x) \bar{\eta} \mathcal{S}^{\mu}\left(x_{1}\right) \mathcal{J}^{\nu}\left(x_{2}\right)\right\rangle\right\rangle= \\
= & \frac{1}{2} \delta^{4}\left(x_{1}, x\right)\left\langle\left\langle\left[K_{\kappa}\left(\mathcal{T}^{\mu \kappa}-G^{\kappa} \mathcal{J}^{\mu}+\frac{1}{2} \varepsilon^{\mu \kappa \rho \lambda} \mathcal{F}_{\rho \lambda}\right)-\frac{1}{4} \nabla_{\lambda}\left(\varepsilon^{\mu \kappa \lambda \sigma} K_{\kappa} \mathcal{J}_{\sigma}\right)+\left(K^{\rho} A_{\rho}\right) \mathcal{J}^{\mu}\right]\left(x_{1}\right) \mathcal{J}^{\nu}\left(x_{2}\right)\right\rangle\right\rangle \\
& +i \delta^{4}\left(x_{2}, x\right)\left\langle\left\langle\overline{\mathcal{S}}^{\nu} \gamma_{*} \zeta\left(x_{2}\right) \bar{\eta} \mathcal{S}^{\mu}\left(x_{1}\right)\right\rangle\right\rangle+\frac{i}{4} \delta^{4}\left(x_{1}, x\right) \delta^{4}\left(x_{1}, x_{2}\right)\left(K^{\nu}\left\langle\mathcal{J}^{\mu}\right\rangle-K^{\mu}\left\langle\mathcal{J}^{\nu}\right\rangle\right) .
\end{aligned}
$$


Let us integrate this over $x$-space, using (see (3.19))

$$
0=\left\langle\delta_{\zeta}\left(\bar{\eta} \mathcal{S}^{\mu}\left(x_{1}\right) \mathcal{J}^{\nu}\left(x_{2}\right)\right)\right\rangle \Longrightarrow 0=\int \mathrm{d}^{4} x \boldsymbol{e} \nabla_{\rho}\left\langle\left\langle\overline{\mathcal{S}}^{\rho} \zeta(x) \bar{\eta} \mathcal{S}^{\mu}\left(x_{1}\right) \mathcal{J}^{\nu}\left(x_{2}\right)\right\rangle\right\rangle
$$

and take a covariant divergence with respect to $x_{1}$ to obtain

$$
\begin{aligned}
0 & =\nabla_{\mu} \int \mathrm{d}^{4} x \boldsymbol{e} i \nabla_{\rho}\left\langle\left\langle\overline{\mathcal{S}}^{\rho} \zeta(x) \bar{\eta} \mathcal{S}^{\mu}\left(x_{1}\right) \mathcal{J}^{\nu}\left(x_{2}\right)\right\rangle\right\rangle \\
& =\frac{1}{2}\left(K^{\lambda} A_{\lambda}\right) \nabla_{\mu}\left\langle\left\langle\mathcal{J}^{\mu}\left(x_{1}\right) \mathcal{J}^{\nu}\left(x_{2}\right)\right\rangle\right\rangle-\frac{i}{2} \delta^{4}\left(x_{1}, x_{2}\right) K^{\nu} \nabla_{\rho}\left\langle\mathcal{J}^{\rho}\left(x_{2}\right)\right\rangle \\
& =\frac{K^{\lambda}}{2} \frac{\delta}{i \boldsymbol{e} \delta A_{\nu}\left(x_{2}\right)}\left(A_{\lambda} \nabla_{\mu}\left\langle\mathcal{J}^{\mu}\right\rangle\right) .
\end{aligned}
$$

For the second equality we used the relation (4.11) (where the first term on the r.h.s. vanishes due to the assumption of the supersymmetric vacuum) and

$$
\begin{aligned}
0=i \nabla_{\mu} & {\left[K_{\nu}\left(\left\langle\left\langle\mathcal{T}^{\mu \nu}\left(x_{1}\right) \mathcal{J}^{\rho}\left(x_{2}\right)\right\rangle\right\rangle+\frac{3}{2}\left\langle\left\langle\mathcal{J}^{\mu}\left(x_{1}\right) \mathcal{J}^{\rho}\left(x_{2}\right)\right\rangle\right\rangle V^{\nu}+\frac{1}{2} \varepsilon^{\mu \nu \rho \lambda}\left\langle\left\langle\mathcal{F}_{\rho \lambda}\left(x_{1}\right) \mathcal{J}^{\rho}\left(x_{2}\right)\right\rangle\right\rangle\right)\right] } \\
& -i K^{\nu} A_{\nu} \nabla_{\mu}\left\langle\left\langle\mathcal{J}^{\mu}\left(x_{1}\right) \mathcal{J}^{\rho}\left(x_{2}\right)\right\rangle\right\rangle-\delta^{4}\left(x_{1}, x_{2}\right)\left[\nabla_{\nu}\left(K^{\nu}\left\langle\mathcal{J}^{\rho}\right\rangle\right)-\left\langle\mathcal{J}^{\nu}\right\rangle \nabla_{\nu} K^{\rho}\right] \\
& +e^{-1} \partial_{\nu}\left[\delta^{4}\left(x_{1}-x_{2}\right)\left(K^{\nu}\left\langle\mathcal{J}^{\rho}\right\rangle-K^{\rho}\left\langle\mathcal{J}^{\nu}\right\rangle\right)\right]
\end{aligned}
$$

which is obtained from (4.1) by replacing $\xi^{\nu}$ by the Killing vector $K^{\nu}$ and using the Killing equations (2.10). Note that in the above computation there occurs a complete cancellation between the contact terms. Using (3.6), (5.7) implies

$$
K^{\lambda} \frac{\delta}{i \boldsymbol{e} \delta A_{\nu}\left(x_{2}\right)}\left(A_{\lambda} \mathcal{A}_{\text {chiral }}\left(x_{1}\right)\right)=0,
$$

which is another constraint in addition to (5.2).

The analysis up to now is insufficient to say about the quantum inconsistency with the known $\mathrm{U}(1)_{R}$ anomaly, because there may exist very restrictive backgrounds on which the constraints (5.2) and (5.9) are satisfied. Therefore we need to go further to higher-point functions. To this end, we differentiate (5.4) once more with respect to the gauge field source $A_{\lambda}\left(x_{3}\right)$ to obtain

$$
\begin{aligned}
i \mathcal{D}_{\rho}\left\langle\left\langle\overline{\mathcal{S}}^{\rho} \epsilon(x) \mathcal{S}^{\mu}\left(x_{1}\right) \mathcal{J}^{\nu}\left(x_{2}\right) \mathcal{J}^{\lambda}\left(x_{3}\right)\right\rangle\right\rangle+\frac{1}{2}\left\langle\left\langle\overline{\mathcal{S}}^{\kappa} \gamma_{\kappa} V^{\rho} \gamma_{\rho} \gamma_{*} \epsilon(x) \mathcal{S}^{\mu}\left(x_{1}\right) \mathcal{J}^{\nu}\left(x_{2}\right) \mathcal{J}^{\lambda}\left(x_{3}\right)\right\rangle\right\rangle= \\
=\frac{1}{2} \delta^{4}\left(x, x_{1}\right) \gamma_{\rho} \epsilon\left\langle\left\langle\left[\mathcal{T}^{\mu \rho}+\frac{3}{4}\left(V^{\rho} \mathcal{J}^{\mu}+V^{\mu} \mathcal{J}^{\rho}-g^{\mu \rho} V_{\kappa} \mathcal{J}^{\kappa}-\varepsilon^{\mu \rho \sigma \kappa} \nabla_{\sigma} \mathcal{J}_{\kappa}\right)+\right.\right.\right. \\
\left.\left.\left.+\frac{1}{2} \varepsilon^{\mu \rho \sigma \kappa} \mathcal{F}_{\sigma \kappa}\right]\left(x_{1}\right) \mathcal{J}^{\nu}\left(x_{2}\right) \mathcal{J}^{\lambda}\left(x_{3}\right)\right\rangle\right\rangle \\
\quad+i \delta^{4}\left(x, x_{2}\right)\left\langle\left\langle\overline{\mathcal{S}}^{\nu} \gamma_{*} \epsilon(x) \mathcal{S}^{\mu}\left(x_{1}\right) \mathcal{J}^{\lambda}\left(x_{3}\right)\right\rangle\right\rangle+i \delta^{4}\left(x, x_{3}\right)\left\langle\left\langle\overline{\mathcal{S}}^{\lambda} \gamma_{*} \epsilon(x) \mathcal{S}^{\mu}\left(x_{1}\right) \mathcal{J}^{\nu}\left(x_{2}\right)\right\rangle\right\rangle \\
+\frac{i}{4} e^{-1} \partial_{\rho}\left[\delta^{4}\left(x-x_{1}\right)\left(-i \varepsilon^{\rho \mu}{ }_{\sigma \kappa} \gamma^{\kappa}+\delta_{\sigma}^{\mu} \gamma_{*} \gamma^{\rho}-\delta_{\sigma}^{\rho} \gamma_{*} \gamma^{\mu}\right) \epsilon\left\langle\left\langle\mathcal{J}^{\sigma}\left(x_{1}\right) \mathcal{J}^{\nu}\left(x_{2}\right) \mathcal{J}^{\lambda}\left(x_{3}\right)\right\rangle\right]\right. \\
\quad-\delta^{4}\left(x, x_{1}\right) \frac{i}{4}\left(-i \varepsilon^{\rho \mu}{ }_{\sigma \kappa} \gamma^{\kappa}+\delta_{\sigma}^{\mu} \gamma_{*} \gamma^{\rho}-\delta_{\sigma}^{\rho} \gamma_{*} \gamma^{\mu}\right) \mathcal{D}_{\rho}\left(\epsilon\left\langle\left\langle\mathcal{J}^{\sigma}\left(x_{1}\right) \mathcal{J}^{\nu}\left(x_{2}\right) \mathcal{J}^{\lambda}\left(x_{3}\right)\right\rangle\right\rangle\right)
\end{aligned}
$$




$$
\begin{aligned}
& +\frac{3 i}{8} \delta^{4}\left(x, x_{1}\right) \gamma_{*} \gamma_{\rho} \epsilon \varepsilon^{\mu \rho \kappa \sigma} V_{\sigma}\left\langle\left\langle\mathcal{J}_{\kappa}\left(x_{1}\right) \mathcal{J}^{\nu}\left(x_{2}\right) \mathcal{J}^{\lambda}\left(x_{3}\right)\right\rangle\right\rangle \\
& -\frac{i}{4} \delta^{4}\left(x, x_{1}\right) \delta^{4}\left(x, x_{2}\right)\left(-i \varepsilon_{\sigma \kappa}^{\nu \mu} \gamma^{\kappa}+\delta_{\sigma}^{\mu} \gamma_{*} \gamma^{\nu}-\delta_{\sigma}^{\nu} \gamma_{*} \gamma^{\mu}\right) \gamma_{*} \epsilon\left\langle\left\langle\mathcal{J}^{\sigma}(x) \mathcal{J}^{\lambda}\left(x_{3}\right)\right\rangle\right\rangle \\
& -\frac{i}{4} \delta^{4}\left(x, x_{1}\right) \delta^{4}\left(x, x_{3}\right)\left(-i \varepsilon_{\sigma \kappa}^{\lambda \mu} \gamma^{\kappa}+\delta_{\sigma}^{\mu} \gamma_{*} \gamma^{\lambda}-\delta_{\sigma}^{\lambda} \gamma_{*} \gamma^{\mu}\right) \gamma_{*} \epsilon\left\langle\left\langle\mathcal{J}^{\sigma}(x) \mathcal{J}^{\nu}\left(x_{2}\right)\right\rangle\right\rangle .
\end{aligned}
$$

By following essentially the same steps as done to reach (5.9) from (5.4) for the 3-point function $\left\langle\left\langle\overline{\mathcal{S}}^{\rho} \epsilon(x) \mathcal{S}^{\mu}\left(x_{1}\right) \mathcal{J}^{\nu}\left(x_{2}\right)\right\rangle\right\rangle$, one can obtain

$$
\begin{aligned}
0= & \nabla_{\mu} \int \mathrm{d}^{4} x \boldsymbol{e} i \nabla_{\rho}\left\langle\left\langle\overline{\mathcal{S}}^{\rho} \zeta(x) \bar{\eta} \mathcal{S}^{\mu}\left(x_{1}\right) \mathcal{J}^{\nu}\left(x_{2}\right) \mathcal{J}^{\lambda}\left(x_{3}\right)\right\rangle\right\rangle \\
= & \frac{1}{2}\left(K^{\rho} A_{\rho}\right) \nabla_{\mu}\left\langle\left\langle\mathcal{J}^{\mu}\left(x_{1}\right) \mathcal{J}^{\nu}\left(x_{2}\right) \mathcal{J}^{\lambda}\left(x_{3}\right)\right\rangle\right\rangle-\frac{i}{2} \delta^{4}\left(x_{1}, x_{2}\right) K^{\nu} \nabla_{\mu}\left\langle\left\langle\mathcal{J}^{\mu}\left(x_{1}\right) \mathcal{J}^{\lambda}\left(x_{3}\right)\right\rangle\right\rangle \\
& -\frac{i}{2} \delta^{4}\left(x_{1}, x_{3}\right) K^{\lambda} \nabla_{\mu}\left\langle\left\langle\mathcal{J}^{\mu}\left(x_{1}\right) \mathcal{J}^{\nu}\left(x_{2}\right)\right\rangle\right\rangle \\
= & \frac{K^{\rho}}{2} \frac{\delta}{i \boldsymbol{e} \delta A_{\lambda}\left(x_{3}\right)} \frac{\delta}{i \boldsymbol{e} \delta A_{\nu}\left(x_{2}\right)}\left(A_{\rho} \nabla_{\mu}\left\langle\mathcal{J}^{\mu}\left(x_{1}\right)\right\rangle\right) \\
= & \frac{K^{\rho}}{2} \frac{\delta}{i \boldsymbol{e} \delta A_{\lambda}\left(x_{3}\right)} \frac{\delta}{i \boldsymbol{e} \delta A_{\nu}\left(x_{2}\right)}\left(A_{\rho} \mathcal{A}_{\text {chiral }}\left(x_{1}\right)\right) .
\end{aligned}
$$

The above procedures can be straightforwardly extended to the higher-point functions obtained by differentiating (5.4) successively with respect to the gauge fields, giving rise to constraints (putting together (5.2), (5.9) and (5.11) here)

$$
\begin{aligned}
0 & =K^{\rho} A_{\rho} \mathcal{A}_{\text {chiral }}(x), \\
0 & =K^{\rho} \frac{\delta}{\boldsymbol{e} \delta A_{\nu}\left(x_{1}\right)}\left(A_{\rho} \mathcal{A}_{\text {chiral }}(x)\right) \\
0 & =K^{\rho} \frac{\delta}{\boldsymbol{e} \delta A_{\lambda}\left(x_{2}\right)} \frac{\delta}{\boldsymbol{e} \delta A_{\nu}\left(x_{1}\right)}\left(A_{\rho} \mathcal{A}_{\text {chiral }}(x)\right), \\
0 & =K^{\rho} \frac{\delta}{\boldsymbol{e} \delta A_{\alpha}\left(x_{3}\right)} \frac{\delta}{\boldsymbol{e} \delta A_{\lambda}\left(x_{2}\right)} \frac{\delta}{\boldsymbol{e} \delta A_{\nu}\left(x_{1}\right)}\left(A_{\rho} \mathcal{A}_{\text {chiral }}(x)\right), \\
0 & =K^{\rho} \frac{\delta}{\boldsymbol{e} \delta A_{\beta}\left(x_{4}\right)} \frac{\delta}{\boldsymbol{e} \delta A_{\alpha}\left(x_{3}\right)} \frac{\delta}{\boldsymbol{e} \delta A_{\lambda}\left(x_{2}\right)} \frac{\delta}{\boldsymbol{e} \delta A_{\nu}\left(x_{1}\right)}\left(A_{\rho} \mathcal{A}_{\text {chiral }}(x)\right)
\end{aligned}
$$

and so on. These constraints are consequences of the Ward identities (3.4)-(3.6) and relation (3.19) with the supersymmetric vacuum condition (5.1). Constraint (5.16) and the subsequent ones with the higher functional derivatives are trivially satisfied, since $\mathcal{A}_{\text {chiral }}$ is quadratic in the gauge field, while (5.12)-(5.15) are nontrivial constraints. In particular, constraint (5.15) with (3.8) gives

$$
\begin{aligned}
0 & =K^{\rho} \frac{\delta}{\boldsymbol{e} \delta A_{\alpha}\left(x_{3}\right)} \frac{\delta}{\boldsymbol{e} \delta A_{\lambda}\left(x_{2}\right)} \frac{\delta}{\boldsymbol{e} \delta A_{\nu}\left(x_{1}\right)}\left(A_{\rho} \mathcal{A}_{\text {chiral }}(x)\right) \\
& =2 c_{A}\left[K^{\alpha} \delta^{4}\left(x, x_{3}\right) \varepsilon^{\kappa \lambda \sigma \nu} \partial_{\kappa} \delta^{4}\left(x, x_{2}\right) \partial_{\sigma} \delta^{4}\left(x, x_{1}\right)+(\text { permutations })\right]
\end{aligned}
$$

which can be satisfied if and only if $c_{A}=0$. Thus, the anomaly coefficient $c_{A}$ should vanish. This makes constraints (5.12)-(5.14) satisfied automatically. 


\section{Discussions}

In this note we have studied the quantum consistency of the new-minimal supergravity formulation of the $\mathcal{N}=1$ supersymmetric theories with an $R$-symmetry in $3+1$ dimensional curved space. By investigating the rigid supersymmetry algebra and the correlation functions obtained via differentiation of the Ward identities (with respect to the background gravitino and $R$-gauge fields), we have shown that the pure $\mathrm{U}(1)_{R}$ chiral anomaly coefficient $c_{A}$ should vanish to be consistent with the supersymmetry. Our result indicates that the supersymmetry is broken at the quantum level unless $c_{A}=0$.

We emphasize that the anomaly coefficient $c_{A}$ depends only on the field content of the theory. There exist some special cases where $c_{A}=0$. For instance, in the $\mathcal{N}=1$ superconformal theories, the anomaly coefficient $c_{A}$ becomes

$$
c_{A}=5 a-3 c,
$$

where the central charges $a$ and $c$ (for free theory) is given by [28, 29]

$$
a=\frac{1}{48}\left(9 N_{V}+N_{\chi}\right), \quad c=\frac{1}{24}\left(3 N_{V}+N_{\chi}\right) .
$$

Here $N_{V}$ and $N_{\chi}$ are the number of gauge and chiral multiplets, respectively. Therefore, $c_{A}$ becomes vanishing when $27 N_{V}=N_{\chi}$, for example when $N_{V}=1$ and $N_{\chi}=27$. Another simple example for a theory with $c_{A}=0$ is the system that consists of a free chiral multiplet with $R$-charge 1 (see e.g. appendix B). In this case, the Weyl fermion in the chiral multiplet is actually uncharged under the $\mathrm{U}(1)_{R}$ symmetry and thus does not contribute to the $\mathrm{U}(1)_{R}$ chiral anomaly.

In this work we have focused on the quantum consistency with respect to the pure $\mathrm{U}(1)_{R}$ anomaly. We expect a similar result for the mixed $\mathrm{U}(1)_{R}$ anomaly coefficient $c_{m}$. Namely, we anticipate that higher-point correlation functions involving both of the supercurrent and the stress-energy tensor can be consistent only when $c_{m}=0$. But it needs more involved computations to show this, which we leave for the future work.

We have assumed that the supersymmetry Ward identity is non-anomalous, see (3.4), and our results bring forward a question about the supersymmetry anomaly, which is also related to the holography. In [30, 31] the holographic renormalization [32-41] was carried out for both of the bosonic and fermionic sector of the $5 \mathrm{D} \mathcal{N}=2$ gauged supergravity, a virtual candidate for holographic dual of $4 \mathrm{D} \mathcal{N}=1$ superconformal field theory (that has an $R$-symmetry). By doing so, it was derived that the supersymmetric Ward identities for $4 \mathrm{D} \mathcal{N}=1$ superconformal field theories (SCFTs) contain anomaly-terms, which lead to the anomalous variation law of supercurrent operators under the rigid supersymmetry transformation. $^{8}$ If one assumes that the supersymmetry Ward identity (3.4) receives anomaly corrections, those anomaly terms would introduce additional (contact) terms in the correlations functions considered in section 4 and 5, which in turn may lead to complete

\footnotetext{
${ }^{8}$ Recently, a similar result was obtained in [42], where it was shown in the context of $\mathrm{AdS}_{3} / \mathrm{CFT}_{2}$ that in $2 \mathrm{D} \mathcal{N}=(1,1)$ superconformal field theories the supercurrent operator transforms anomalously under the rigid supersymmetry transformation.
} 
cancellations on the right-hand sides of (5.12)-(5.16) restoring the consistency of the theory with $c_{A} \neq 0 .{ }^{9}$ It would be interesting to explore if the anomaly corrections obtained in $[30,31]$ by holographic renormalization could do the job (see $[43,44]$ for recent fieldtheoretical studies on the supersymmetry anomalies). We hope to pursue this question in the future work.

\section{Acknowledgments}

We thank Gwang Il Kim and Ui Ri Mun for useful discussions and comments. This research was supported in part by NSTC Project No. 130-01.

\section{A Conventions}

We follow the conventions used in [45]. The metric signature is $(-,+,+,+)$ and $\varepsilon_{0123}=1$. We denote $\gamma_{5}$ matrix by $\gamma_{*}$ in 4 dimensions, i.e.

$$
\gamma_{*}=i \gamma_{0} \gamma_{1} \gamma_{2} \gamma_{3}
$$

which leads to useful formulas

$$
\begin{aligned}
\gamma^{\mu \nu \rho} \gamma_{*} & =i \varepsilon^{\mu \nu \rho \sigma} \gamma_{\sigma}, \\
\varepsilon^{\mu \nu \rho \lambda} \gamma_{\rho \lambda} & =-2 i \gamma^{\mu \nu} \gamma_{*} .
\end{aligned}
$$

For any spinor $\chi, \bar{\chi} \equiv \chi^{T} C$ is the Majorana conjugate of $\chi$, where $C$ is the charge conjugation matrix. And all of the spinors in this note are Majorana ones.

In our conventions,

$$
\begin{aligned}
\bar{\lambda} \Gamma^{\left(r_{1}\right)} \cdots \Gamma^{\left(r_{p}\right)} \chi & =t_{0}^{p-1} t_{r_{1}} \cdots t_{r_{p}} \bar{\chi} \Gamma^{\left(r_{p}\right)} \cdots \Gamma^{\left(r_{1}\right)} \lambda, \\
\chi & =\Gamma^{\left(r_{1}\right)} \cdots \Gamma^{\left(r_{p}\right)} \lambda \Longrightarrow \bar{\chi}=t_{0}^{p} t_{r_{1}} \cdots t_{r_{p}} \bar{\lambda} \Gamma^{\left(r_{p}\right)} \cdots \Gamma^{\left(r_{1}\right)},
\end{aligned}
$$

where

$$
t_{0}=t_{3}=1, \quad t_{1}=t_{2}=-1 .
$$

Two kinds of connections appear in this note, i.e. the metric connection and the gauge connection. The symbol $\nabla$ stands for the covariant derivative with respect to the metric. The symbol $\mathcal{D}$ indicates the connection with respect to the metric and the gauge field. For instance,

$$
\mathcal{D}_{\mu} \psi_{\nu} \equiv \nabla_{\mu} \psi_{\nu}+i G_{\mu} \gamma_{*} \psi_{\nu}, \quad \mathcal{D}_{\mu} \bar{\psi}_{\nu}=\nabla_{\mu} \bar{\psi}_{\nu}+i G_{\mu} \bar{\psi}_{\nu} \gamma_{*},
$$

\footnotetext{
${ }^{9}$ One relevant question is whether there could exist local counterterms added to the action in such a way that the consistency of the theory is maintained without requiring $c_{A}=0$. In order for the local counterterms to change the argument of this paper and restore the consistency of the theory with $c_{A} \neq 0$, they should modify the Ward identities (3.4)-(3.6) by introducing appropriate additional local terms to the Ward identities. However, the local counterterms that modify the Ward identities necessarily break the corresponding symmetries. Therefore, as far as one does not want to break supersymmetry and diffeomorphism invariance explicitly, we expect that local counterterms can not play a role in retrieving the consistency of the theory with $c_{A} \neq 0$.
} 
where $G_{\mu} \equiv A_{\mu}-\frac{3}{2} V_{\mu}$. Note that the supercurrent $\mathcal{S}^{\mu}$ is conjugate to the source $\psi_{\mu}$, and therefore it has a $\mathrm{U}(1)_{R}$ charge opposite to $\psi_{\mu}$. It follows that one has to define the covariant derivative of $\mathcal{S}^{\mu}$ by

$$
\mathcal{D}_{\mu} \mathcal{S}^{\nu}=\nabla_{\mu} \mathcal{S}^{\nu}-i G_{\mu} \gamma_{*} \mathcal{S}^{\nu}
$$

\section{B An example: $\mathcal{N}=1$ with a free chiral multiplet on $\mathbb{R} \times S_{r_{3}}$}

In this appendix we explicitly derive the variation of the current operators $\mathcal{S}^{\mu}, \mathcal{J}^{\mu}$ and $\mathcal{F}^{\mu \nu}$ under the rigid supersymmetry transformation, for the special case when $\mathcal{N}=1$ field theory for a free chiral multiplet is defined on $\mathbb{R} \times S_{r_{3}}$.

The action of the theory that we are interested in is given by [6],

$$
S=\int \mathrm{d}^{4} x \mathcal{L}
$$

where

$\frac{1}{e} \mathcal{L}=F F^{*}-D_{\mu} \phi D^{\mu} \phi^{*}+i V^{\mu}\left(\phi^{*} D_{\mu} \phi-\phi D_{\mu} \phi^{*}\right)-\frac{q}{4}\left(R+6 V_{\mu} V^{\mu}\right) \phi^{*} \phi-\bar{\psi} \gamma^{\mu}\left(D_{\mu}-\frac{i}{2} V_{\mu}\right) P_{L} \psi$,

and $q$ is the $\mathrm{U}(1)_{R^{-}}$-charge of the chiral multiplet, and

$$
\begin{aligned}
R & =\frac{6}{r^{2}}, \quad V_{i}=0, V_{t}=\frac{1}{r}, \quad A_{t}=\frac{1}{r}, A_{i}=0, \quad R_{\mu \nu}=2\left(V_{\mu} V_{\nu}-g_{\mu \nu} V_{\rho} V^{\rho}\right), \\
D_{\mu} \phi & \equiv \partial_{\mu} \phi-i q A_{\mu} \phi, \quad D_{\mu} \phi^{*} \equiv \partial_{\mu} \phi+i q A_{\mu} \phi, \quad D_{\mu} \psi \equiv \nabla_{\mu} \psi+i(q-1) A_{\mu} \gamma_{*} \psi, \\
\nabla_{\mu} \psi & \equiv \partial_{\mu} \psi+\frac{1}{4} \omega_{\mu a b} \gamma^{a b} \psi, \quad P_{L, R} \equiv \frac{1 \mp \gamma_{*}}{2} .
\end{aligned}
$$

This theory is invariant under the rigid supersymmetry

$$
\begin{aligned}
\delta \phi & =\bar{\zeta} P_{L} \psi, & \delta \phi^{*} & =\bar{\zeta} P_{R} \psi, \\
\delta F & =\bar{\zeta} \gamma^{\mu}\left(D_{\mu}-\frac{i}{2} V_{\mu}\right) P_{L} \psi, & \delta F^{*} & =\bar{\zeta} \gamma^{\mu}\left(D_{\mu}+\frac{i}{2} V_{\mu}\right) P_{R} \psi, \\
\delta P_{L} \psi & =P_{L}(\not D \phi+F) \epsilon, & \delta P_{R} \psi & =P_{R}\left(\not D \phi^{*}+F^{*}\right) \epsilon,
\end{aligned}
$$

where $\zeta$ is the GKS that satisfies the GKS condition (2.7). Note that under the charge conjugation $\gamma_{*}$ flips the sign and thus $\left(P_{L}\right)^{C}=P_{R}$.

The energy-momentum tensor is given by

$$
\begin{aligned}
\mathcal{T}^{\mu \nu}= & -\frac{1}{2 \boldsymbol{e}}\left(e^{a \nu} \frac{\delta}{\delta e_{\mu}^{a}}+e^{a \mu} \frac{\delta}{\delta e_{\nu}^{a}}\right) \mathcal{S} \\
= & -g^{\mu \nu}\left[-D_{\rho} \phi D^{\rho} \phi^{*}+F F^{*}-\frac{q}{4}\left(R-6 V^{\rho} V_{\rho}\right) \phi^{*} \phi\right]-2 D^{(\mu} \phi D^{\nu)} \phi^{*}+ \\
& +\frac{q}{2}\left(-R^{\mu \nu}+\nabla^{\mu} \nabla^{\nu}-g^{\mu \nu} \square+6 V^{\mu} V^{\nu}\right)\left(\phi^{*} \phi\right)-\frac{i}{2} V^{(\mu} \bar{\psi} \gamma^{\nu)} \psi \\
& +\frac{1}{2}\left[g^{\mu \nu} \bar{\psi} \gamma^{\rho} \overleftrightarrow{D}_{\rho} \psi-\bar{\psi} \gamma^{(\mu} \overleftrightarrow{D^{\nu}} \psi\right]
\end{aligned}
$$


and the $\mathrm{U}(1)_{R^{-} \text {-current is }}$

$$
\mathcal{J}^{\mu}=\frac{1}{e} \frac{\delta S}{\delta A_{\mu}}=i q\left(\phi D^{\mu} \phi^{*}-\phi^{*} D^{\mu} \phi\right)+2 q V^{\mu} \phi \phi^{*}+i(q-1) \bar{\psi} \gamma^{\mu} P_{L} \psi
$$

Since the Lagrangian (B.2) does not possess any FI-terms and the Kähler form of the target space is exact, there exists a well-defined operator $Y_{\mu}$, such that $\mathcal{F}_{\mu \nu}=\partial_{\mu} Y_{\nu}-\partial_{\nu} Y_{\mu}$, see e.g. [12]. Defining an operator $\mathcal{K}^{\mu}$ by

$$
\mathcal{K}^{\mu} \equiv \frac{1}{\boldsymbol{e}} \frac{\delta S}{\delta V_{\mu}}=i\left(\phi^{*} D^{\mu} \phi-\phi D^{\mu} \phi^{*}\right)-3 q V^{\mu} \phi^{*} \phi+\frac{i}{2} \bar{\psi} \gamma^{\mu} P_{L} \psi
$$

we have an operator relation

$$
Y^{\mu}=K^{\mu}+\frac{3}{2} \mathcal{J}^{\mu}=\frac{i}{2}(3 q-2)\left(\phi D^{\mu} \phi^{*}-\phi^{*} D^{\mu} \phi+\bar{\psi} \gamma^{\mu} P_{L} \psi\right)
$$

because

$$
\delta V_{\mu}=\frac{1}{4} \varepsilon_{\mu}{ }^{\nu \lambda} \partial_{\nu} \delta B_{\rho \lambda}+\left(\delta g_{\mu \nu}\right) V^{\nu}-\frac{1}{2} g^{\rho \lambda} \delta g_{\rho \lambda} V_{\mu} .
$$

Notice that when $q=2 / 3$ the operator $Y^{\mu}$ and $\mathcal{F}_{\mu \nu}$ are identically vanishing. Since the conformal symmetry is explicitly broken by the operator $\mathcal{F}_{\mu \nu}[8]$, this implies that the theory becomes superconformal.

We could find the supercurrent by obtaining the Noether current corresponding to the transformation given by (B.6). Instead, we would like to use (4.17) to find the supercurrent, which would differ from the Noether current by a term like $D_{\nu} \mathcal{M}^{\mu \nu}$. The variation of the $R$-current $\mathcal{J}^{\mu}$ is

$$
\begin{aligned}
\delta_{\zeta} \mathcal{J}^{\mu}=-i \bar{\zeta}(\not D \phi) & \gamma^{\mu} P_{R} \psi+i q D_{\nu}(\phi \bar{\psi}) \gamma^{\mu \nu} P_{R} \zeta+\frac{3 q}{2} \phi V^{\mu} \bar{\psi} P_{R} \zeta \\
& +i q \phi \bar{\zeta} \gamma^{\mu} \gamma^{\nu}\left[D_{\nu}\left(P_{R} \psi\right)+\frac{i}{2} V_{\nu} P_{R} \psi\right]+i(q-1) \bar{\psi} \gamma^{\mu} P_{L} \zeta F+\text { h.c. }
\end{aligned}
$$

so we find that

$$
\mathcal{S}^{\mu}=-i \bar{\zeta}(\not D \phi) \gamma^{\mu} P_{R} \psi+i q D_{\nu}(\phi \bar{\psi}) \gamma^{\mu \nu} P_{R} \zeta+\frac{3 q}{2} \phi V^{\mu} \bar{\psi} P_{R} \zeta+\text { h.c. }
$$

where we used the equations of motion of the theory. Notice that although the rigid supersymmetry algebra is off-shell, the transformation rules (4.17), (4.18) and (4.10) should be on-shell relations at the classical level, as we see below.

The Gamma trace of the supercurrent (B.12) is

$$
\gamma_{\mu} \mathcal{S}^{\mu}=-(3 q-2)(\not D \phi) P_{R} \psi-3 q \phi \gamma^{\nu}\left[D_{\nu}\left(P_{R} \psi\right)+\frac{i}{2} V_{\nu} P_{R} \psi\right]+\text { h.c. } .
$$

This vanishes on-shell for $q=2 / 3$, which is related to the fact that the theory has the superconformal symmetry when $q=2 / 3$. It is also implied by (B.13) that (4.18) cannot hold off-shell, since $Y_{\mu}$ vanishes identically for $q=2 / 3$. 
One can see that (4.18) holds on-shell by observing that

$$
\begin{aligned}
\delta_{\zeta} Y^{\mu} & =\frac{i}{2}(3 q-2)\left[\phi D^{\mu}\left(\bar{\zeta} P_{R} \psi\right)-\bar{\zeta} P_{R} \psi D^{\mu} \phi+\bar{\psi} \gamma^{\mu} P_{L}(\not D \phi+F) \zeta\right]+\text { h.c. } \\
& =\frac{i}{2}(3 q-2)\left[D^{\mu}\left(\phi \bar{\zeta} P_{R} \psi\right)-\bar{\zeta} \not D \phi \gamma^{\mu} P_{R} \psi+\bar{\psi} \gamma^{\mu} P_{L} \zeta\right]+\text { h.c. }
\end{aligned}
$$

By a tedious computation it can be explicitly shown that the transformation law for (4.10) also holds on-shell.

We emphasize that the whole analysis in this appendix can be extended to the more general backgrounds that admit two supercharges with opposite $R$-charge.

Open Access. This article is distributed under the terms of the Creative Commons Attribution License (CC-BY 4.0), which permits any use, distribution and reproduction in any medium, provided the original author(s) and source are credited.

\section{References}

[1] N.A. Nekrasov, Seiberg-Witten prepotential from instanton counting, Adv. Theor. Math. Phys. 7 (2003) 831 [hep-th/0206161] [InSPIRE].

[2] V. Pestun, Localization of gauge theory on a four-sphere and supersymmetric Wilson loops, Commun. Math. Phys. 313 (2012) 71 [arXiv:0712.2824] [INSPIRE].

[3] J.M. Maldacena, The Large $N$ limit of superconformal field theories and supergravity, Int. J. Theor. Phys. 38 (1999) 1113 [hep-th/9711200] [INSPIRE].

[4] E. Witten, Anti-de Sitter space and holography, Adv. Theor. Math. Phys. 2 (1998) 253 [hep-th/9802150] [INSPIRE].

[5] S.S. Gubser, I.R. Klebanov and A.M. Polyakov, Gauge theory correlators from noncritical string theory, Phys. Lett. B 428 (1998) 105 [hep-th/9802109] [INSPIRE].

[6] G. Festuccia and N. Seiberg, Rigid Supersymmetric Theories in Curved Superspace, JHEP 06 (2011) 114 [arXiv:1105.0689] [INSPIRE].

[7] T.T. Dumitrescu, G. Festuccia and N. Seiberg, Exploring Curved Superspace, JHEP 08 (2012) 141 [arXiv: 1205.1115] [INSPIRE].

[8] C. Klare, A. Tomasiello and A. Zaffaroni, Supersymmetry on Curved Spaces and Holography, JHEP 08 (2012) 061 [arXiv: 1205.1062] [INSPIRE].

[9] T.T. Dumitrescu, An introduction to supersymmetric field theories in curved space, J. Phys. A 50 (2017) 443005 [arXiv:1608.02957] [InSPIRE].

[10] M.F. Sohnius and P.C. West, An Alternative Minimal Off-Shell Version of $N=1$ Supergravity, Phys. Lett. B 105 (1981) 353 [INSPIRE].

[11] M. Sohnius and P.C. West, The Tensor Calculus and Matter Coupling of the Alternative Minimal Auxiliary Field Formulation of $N=1$ Supergravity, Nucl. Phys. B 198 (1982) 493 [INSPIRE].

[12] C. Closset, T.T. Dumitrescu, G. Festuccia and Z. Komargodski, The Geometry of Supersymmetric Partition Functions, JHEP 01 (2014) 124 [arXiv:1309.5876] [INSPIRE]. 
[13] C. Closset, T.T. Dumitrescu, G. Festuccia and Z. Komargodski, From Rigid Supersymmetry to Twisted Holomorphic Theories, Phys. Rev. D 90 (2014) 085006 [arXiv:1407.2598] [INSPIRE].

[14] B. Assel, D. Cassani and D. Martelli, Localization on Hopf surfaces, JHEP 08 (2014) 123 [arXiv: 1405.5144] [INSPIRE].

[15] D. Cassani and D. Martelli, Supersymmetry on curved spaces and superconformal anomalies, JHEP 10 (2013) 025 [arXiv: 1307.6567] [INSPIRE].

[16] B. Assel, D. Cassani, L. Di Pietro, Z. Komargodski, J. Lorenzen and D. Martelli, The Casimir Energy in Curved Space and its Supersymmetric Counterpart, JHEP 07 (2015) 043 [arXiv: 1503.05537] [INSPIRE].

[17] D. Cassani and D. Martelli, The gravity dual of supersymmetric gauge theories on a squashed $S^{1} \times S^{3}$, JHEP 08 (2014) 044 [arXiv: 1402.2278] [INSPIRE].

[18] S.J. Gates Jr., M.T. Grisaru and W. Siegel, Auxiliary Field Anomalies, Nucl. Phys. B 203 (1982) 189 [inSPIRE].

[19] S.J. Gates Jr., M.T. Grisaru, M. Roček and W. Siegel, Superspace Or One Thousand and One Lessons in Supersymmetry, Front. Phys. 58 (1983) 1 [hep-th/0108200] [INSPIRE].

[20] P. Benetti Genolini, D. Cassani, D. Martelli and J. Sparks, The holographic supersymmetric Casimir energy, Phys. Rev. D 95 (2017) 021902 [arXiv:1606. 02724] [INSPIRE].

[21] P. Benetti Genolini, D. Cassani, D. Martelli and J. Sparks, Holographic renormalization and supersymmetry, JHEP 02 (2017) 132 [arXiv:1612.06761] [INSPIRE].

[22] D. Cassani, C. Klare, D. Martelli, A. Tomasiello and A. Zaffaroni, Supersymmetry in Lorentzian Curved Spaces and Holography, Commun. Math. Phys. 327 (2014) 577 [arXiv: 1207.2181] [INSPIRE].

[23] H. Osborn, Weyl consistency conditions and a local renormalization group equation for general renormalizable field theories, Nucl. Phys. B 363 (1991) 486 [INSPIRE].

[24] I. Papadimitriou, Lectures on Holographic Renormalization, Springer Proc. Phys. 176 (2016) 131 [INSPIRE].

[25] K. Jensen, R. Loganayagam and A. Yarom, Thermodynamics, gravitational anomalies and cones, JHEP 02 (2013) 088 [arXiv: 1207.5824] [INSPIRE].

[26] P. Di Francesco, P. Mathieu and D. Senechal, Conformal Field Theory, Graduate Texts in Contemporary Physics, Springer-Verlag, New York U.S.A. (1997).

[27] I. Papadimitriou, Holographic renormalization as a canonical transformation, JHEP 11 (2010) 014 [arXiv: 1007.4592] [InSPIRE].

[28] S.M. Christensen and M.J. Duff, Axial and Conformal Anomalies for Arbitrary Spin in Gravity and Supergravity, Phys. Lett. B 76 (1978) 571 [INSPIRE].

[29] D. Anselmi, J. Erlich, D.Z. Freedman and A.A. Johansen, Positivity constraints on anomalies in supersymmetric gauge theories, Phys. Rev. D 57 (1998) 7570 [hep-th/9711035] [INSPIRE].

[30] I. Papadimitriou, Supercurrent anomalies in 4d SCFTs, JHEP 07 (2017) 038 [arXiv: 1703. 04299] [INSPIRE].

[31] O.S. An, Anomaly-corrected supersymmetry algebra and supersymmetric holographic renormalization, JHEP 12 (2017) 107 [arXiv:1703.09607] [INSPIRE]. 
[32] M. Henningson and K. Skenderis, The Holographic Weyl anomaly, JHEP 07 (1998) 023 [hep-th/9806087] [INSPIRE].

[33] V. Balasubramanian and P. Kraus, A Stress tensor for Anti-de Sitter gravity, Commun. Math. Phys. 208 (1999) 413 [hep-th/9902121] [INSPIRE].

[34] J. de Boer, E.P. Verlinde and H.L. Verlinde, On the holographic renormalization group, JHEP 08 (2000) 003 [hep-th/9912012] [INSPIRE].

[35] P. Kraus, F. Larsen and R. Siebelink, The gravitational action in asymptotically AdS and flat space-times, Nucl. Phys. B 563 (1999) 259 [hep-th/9906127] [INSPIRE].

[36] S. de Haro, S.N. Solodukhin and K. Skenderis, Holographic reconstruction of space-time and renormalization in the AdS/CFT correspondence, Commun. Math. Phys. 217 (2001) 595 [hep-th/0002230] [INSPIRE].

[37] M. Bianchi, D.Z. Freedman and K. Skenderis, How to go with an RG flow, JHEP 08 (2001) 041 [hep-th/0105276] [INSPIRE].

[38] M. Bianchi, D.Z. Freedman and K. Skenderis, Holographic renormalization, Nucl. Phys. B 631 (2002) 159 [hep-th/0112119] [InSPIRE].

[39] D. Martelli and W. Mueck, Holographic renormalization and Ward identities with the Hamilton-Jacobi method, Nucl. Phys. B 654 (2003) 248 [hep-th/0205061] [INSPIRE].

[40] K. Skenderis, Lecture notes on holographic renormalization, Class. Quant. Grav. 19 (2002) 5849 [hep-th/0209067] [INSPIRE].

[41] I. Papadimitriou and K. Skenderis, AdS/CFT correspondence and geometry, IRMA Lect. Math. Theor. Phys. 8 (2005) 73 [hep-th/0404176] [INSPIRE].

[42] O.S. An, Y.H. Ko and S.-H. Won, Super-Weyl Anomaly from Holography and Rigid Supersymmetry Algebra on Two-Sphere, arXiv:1812.10209 [INSPIRE].

[43] G. Katsianis, I. Papadimitriou, K. Skenderis and M. Taylor, Anomalous Supersymmetry, arXiv: 1902.06715 [INSPIRE].

[44] I. Papadimitriou, Supersymmetry anomalies in $\mathcal{N}=1$ conformal supergravity, JHEP 04 (2019) 040 [arXiv:1902.06717] [INSPIRE].

[45] D.Z. Freedman and A. Van Proeyen, Supergravity, Cambridge University Press, Cambridge U.K. (2012).

[46] S.P. Martin, A Supersymmetry primer, Adv. Ser. Direct. High Energy Phys. 21 (2010) 1 [Adv. Ser. Direct. High Energy Phys. 18 (1998) 1] [hep-ph/9709356] [INSPIRE].

[47] J. Wess and J. Bagger, Supersymmetry and supergravity, Princeton University Press, Princeton U.S.A. (1992).

[48] E. Gerchkovitz, Constraints on the R-charges of free bound states from the Römelsberger index, JHEP 07 (2014) 071 [arXiv: 1311.0487] [INSPIRE].

[49] L. Álvarez-Gaumé and E. Witten, Gravitational Anomalies, Nucl. Phys. B 234 (1984) 269 [INSPIRE].

[50] A. Cappelli and A. Coste, On the Stress Tensor of Conformal Field Theories in Higher Dimensions, Nucl. Phys. B 314 (1989) 707 [InSPIRE].

[51] L.S. Brown and J.P. Cassidy, Stress Tensors and their Trace Anomalies in Conformally Flat Space-Times, Phys. Rev. D 16 (1977) 1712 [InSPIRE]. 
[52] E.S. Fradkin and A.A. Tseytlin, Conformal Anomaly in Weyl Theory and Anomaly Free Superconformal Theories, Phys. Lett. B 134 (1984) 187 [InSPIRE].

[53] B. Assel, D. Cassani and D. Martelli, Supersymmetric counterterms from new minimal supergravity, JHEP 11 (2014) 135 [arXiv: 1410.6487] [INSPIRE].

[54] D. Anselmi, D.Z. Freedman, M.T. Grisaru and A.A. Johansen, Nonperturbative formulas for central functions of supersymmetric gauge theories, Nucl. Phys. B 526 (1998) 543 [hep-th/9708042] [INSPIRE].

[55] I. Papadimitriou, Holographic Renormalization of general dilaton-axion gravity, JHEP 08 (2011) 119 [arXiv: 1106.4826] [InSPIRE].

[56] R. Blumenhagen, D. Lüst and S. Theisen, Basic concepts of string theory, Theoretical and Mathematical Physics, Springer (2013). 